UMD-PP-08-014

MADPH-08-1520

UCD-2008-03

\title{
LHC Signals for Warped Electroweak Charged Gauge Bosons
}

\author{
Kaustubh Agashe $^{a}$, Shrihari Gopalakrishna ${ }^{b}$, Tao Han ${ }^{c}$, Gui-Yu Huang $^{c, d}$, Amarjit Soni ${ }^{b}$ \\ ${ }^{a}$ Maryland Center for Fundamental Physics, Department of Physics, University of Maryland, \\ College Park, MD 20742, USA. \\ ${ }^{b}$ Brookhaven National Laboratory, Upton, NY 11973, USA. \\ ${ }^{c}$ Department of Physics, University of Wisconsin, Madison, WI 53706, USA. \\ ${ }^{d}$ Department of Physics, University of California at Davis, Davis, CA 95616, USA.
}

\begin{abstract}
We study signals at the Large Hadron Collider (LHC) for the Kaluza-Klein (KK) excitations of electroweak charged gauge bosons in the framework of the Standard Model (SM) fields propagating in the bulk of a warped extra dimension. Such a scenario can solve both the Planck-weak and flavor hierarchy problems of the SM. There are two such charged states in this scenario with couplings to light quarks and leptons being suppressed relative to those in the SM, whereas the couplings to top/bottom quarks are enhanced, similar to the case of electroweak neutral gauge bosons previously studied. However, unlike the case of electroweak neutral gauge bosons, there is no irreducible QCD background (including pollution from possibly degenerate KK gluons) for decays to top + bottom final state so that this channel is useful for the discovery of the charged states. Moreover, decays of electroweak charged gauge bosons to longitudinal $W, Z$ and Higgs are enhanced just as for the neutral bosons. However, unlike for the neutral gauge bosons, the purely leptonic (and hence clean) decay mode of the $W Z$ are fully reconstructible so that the ratio of the signal to the SM (electroweak) background can potentially be enhanced by restricting to the resonance region more efficiently. We show that such final states can give sensitivity to 2 (3) $\mathrm{TeV}$ masses with an integrated luminosity of $100(300) \mathrm{fb}^{-1}$. We emphasize that improvements in discriminating a QCD-jet from a highly boosted hadronically decaying $W$, and a highly boosted top-jet from a bottom-jet will enhance the reach for these KK particles, and that the signals we study for the warped extra dimensional model might actually be applicable also to a wider class of non-supersymmetric models of electroweak symmetry breaking.
\end{abstract}




\section{Introduction}

The era of the Large Hadron Collider (LHC) is upon us! Experiments at the LHC are highly expected to shed light on the mechanism of electroweak symmetry breaking (EWSB). In particular, various extensions of the Standard Model (SM) have been proposed to solve the problem in the SM of the hierarchy between the Planck and electroweak scales. Such models predict the existence of new particles at the weak (or $\mathrm{TeV}$ ) scale which are likely to be accessible to the LHC.

In the present work, we focus on one such extension of the SM, the Randall-Sundrum model (RS1) [1] with all the SM fields propagating in the bulk of a warped extra dimension [2, 3, 4]. Such a framework can address the flavor hierarchy problem of the SM as well. The versions of this framework with a grand unified gauge symmetry in the bulk can naturally lead to precision unification of the three SM gauge couplings [5] and a candidate for the dark matter of the universe (the latter from requiring longevity of the proton) [6]. The new particles in this framework are Kaluza-Klein $(\mathrm{KK})$ excitations of all SM fields with masses at $\sim \mathrm{TeV}$ scale. So far, studies of the LHC signals from direct production of the radion [7], KK gluon [8, 9, 10, 11], graviton [12], neutral electroweak gauge bosons [13], (heavy) fermions [14] and finally light KK fermions present in some models with extended $5 D$ gauge symmetries [15] in such a framework have been performed (see Ref. [14] for an overview and Refs. [16] for related studies in other set-ups within the warped extra dimensional framework).

However, there are some challenging aspects of this collider phenomenology as follows. Firstly, the KK mass scale is constrained to be at least a few $\mathrm{TeV}$ by the electroweak and flavor precision tests in part due to the absence of a parity symmetry (analogous to $R$-parity in SUSY), allowing tree-level exchanges to contribute to the precision observables. In addition, the constituents of the proton (or SM gauge bosons and light fermions in general) couple weakly to the KK states, whereas the KK states mostly decay to top quarks and longitudinal $W / Z /$ Higgs due to a larger coupling to these states. As a result, the golden decay channels such as resonant signals of dileptons or diphotons are suppressed. Finally, given the few TeV KK mass, the top quarks $/ W / Z$ resulting from the decays of these KK states are highly boosted, creating problems in their identification due to collimation of their decay products.

In light of this situation, it is necessary to study as many LHC probes of this framework as possible, especially since there might not be a single "smoking gun" for this framework, i.e., a variety of channels can complement each other as far as detecting this framework at the LHC is concerned. In particular, the most widely studied particle is the KK gluon which decays only to jetty final states, but has the largest cross-section due to the QCD coupling (assuming the same mass for all KK particles as in the simplest models). It was found that the LHC reach can be $\sim 4$ $\mathrm{TeV}$, using techniques designed specifically to identify highly boosted top quarks. However, it is 
good to have channels with no jets if possible, since in general such modes are cleaner in the LHC experimental environment.

Also, it is obviously important to explore the feasibility of searching for the electroweak (EW) KK states (i.e., excitations of $\gamma, W$ and $Z$ ) at the LHC. In fact, decays of EW KK states to (longitudinal) $W / Z$ and Higgs offer a possibility for clean final states if these SM particles decay to leptons (note that the direct decays to leptons/photons are suppressed as mentioned above). However, the decay of the $\mathrm{KK} Z$ to $W W$ followed by leptonic decays of both $W$ 's has two neutrinos in the final state so that the invariant mass of $W$ pair cannot be effectively reconstructed, making it harder to identify the signal and to reduce the continuum SM $W W$ background. If one $W$ decays instead to a pair of quarks (we call it "semileptonic" decay of the $W W$ gauge boson pair), then the problem is that the two jets from the $W$ are collimated, introducing a larger QCD background from $W+$ jet. A similar analysis applies to decays of the KK $Z$ to the $Z h$ final state. Finally, one could utilize decays to top pairs for detecting the KK $Z$ using techniques to identify boosted tops developed for detecting the KK gluon, but this channel is swamped by decays of the KK gluon to (i.e., resonant production of) top pairs, if not by the SM $t \bar{t}$ continuum background.

Given this situation, the KK $W$ can provide (a priori) a couple of advantages:

- The decays to $W Z$ followed by (clean) leptonic decays of both $W$ and $Z$ can be more effectively reconstructed due to the presence of only one neutrino.

The semileptonic decays of $W Z$ or $W h$ face similar challenges to those of $\mathrm{KK} Z$, namely, QCD $Z / W+$ jet background and we can use a jet mass cut in order to reduce this background, i.e., to distinguish a $W / Z$-jet from a $\mathrm{QCD}$ jet.

- The decays of KK $W$ to $t \bar{b}$ do not have the contamination from the KK gluon as in the case of KK $Z$.

With this background, we are thus motivated to study signals for the KK excitation of the SM $W$ in this paper. With detailed parton-level simulations for the signal and SM backgrounds, we find the reach for this particle to be $2(3) \mathrm{TeV}$ with $\sim 100(300) \mathrm{fb}^{-1}$ luminosity in the $t \bar{b}, W Z$ and $W h$ channels, a discovery potential which is roughly similar to that for the $\mathrm{KK} Z$, found earlier in [13]. The reason for the similar (although slightly better) reach for the KK $W$ as for the $\mathrm{KK} Z$ in spite of the expected above two advantages for the former are that, firstly, the BR to leptons for the SM $Z$ is smaller than that for the $W$, making the final significance of the leptonic decays of the $W Z$ from the KK $W$ not much better than in the case of the purely leptonic decays of the $W$ pairs from the KK $Z$. Secondly, we find that a highly boosted top quark can fake a bottom quark so that QCD or KK gluon $t \bar{t}$ pairs do manifest as reducible backgrounds to $t \bar{b}$ signal from the KK $W$. Once again, we use a jet mass cut, this time to discriminate between a $t$-jet and a $b$-jet. Anticipating more 
dedicated analyses in regard to vetoing $t \bar{t}$ background from KK gluon decays, we believe that it is possible to make the reach in KK $W$ better than KK $Z$ via the $t \bar{b}$ channel, perhaps comparable to KK gluon. Similarly, further improvements in the jet mass technique of distinguishing a $W / Z$-jet from a QCD jet or the development of new ones to reduce this QCD background can increase the reach for both $\mathrm{KK} W$ and $Z$ in semileptonic $W W / W Z$ decays of these KK modes.

The outline of the paper is as follows. In Sec. 2, we begin with a brief review of the theory of a warped extra dimension and the LHC signals for the KK states, including an outline of the various cases we consider for the study of the electroweak charged gauge bosons. We present the total decay widths of $W^{\prime \prime}$ s and the branching ratios to various channels in Sec. 3. In Sec. 4, we calculate the production cross-sections of the charged gauge bosons at the LHC and present a detailed analysis of how to obtain signals for these states. The framework of warped extra dimension is conjectured to be dual to four-dimensional ( $4 D$ ) strong dynamics triggering electroweak symmetry breaking, as in technicolor or composite Higgs models. In Sec. 5 , we then compare the signals that we studied in a warped extra dimension to the signals for technicolor models discussed previously (since 1990's). Further discussions and conclusions are presented in Sec. 6, where we argue that many of the signals that we study here (including the electroweak neutral gauge boson case studied earlier) might be applicable to a wider class of non-supersymmetric models of EWSB. Finally, two Appendices are included at the end to provide further details for the model, including the couplings of the $W^{\prime}$ states.

\section{Review of Warped Extra Dimension}

The framework consists of a slice of anti-de Sitter space in five dimensions $\left(\mathrm{AdS}_{5}\right)$, where (due to the warped geometry) the effective $4 D$ mass scale is dependent on position in the extra dimension. The $4 D$ graviton, i.e., the zero-mode of the $5 D$ graviton, is automatically localized at one end of the extra dimension (called the Planck/UV brane). If the Higgs sector is localized at the other end (in fact with SM Higgs originating as 5 th component of a $5 D$ gauge field $\left(A_{5}\right)$ it is automatically so [17]), then the warped geometry naturally generates the Planck-weak hierarchy. Specifically, TeV

$\sim \bar{M}_{P} e^{-k \pi r_{c}}$, where $\bar{M}_{P}$ is the reduced $4 D$ Planck scale, $k$ is the $\mathrm{AdS}_{5}$ curvature scale and $r_{c}$ is the proper size of the extra dimension. The crucial point is that the required modest size of the radius (in units of the curvature radius), i.e., $k r_{c} \sim 1 / \pi \log \left(\bar{M}_{P} / \mathrm{TeV}\right) \sim 10$ can be stabilized with only a corresponding modest tuning in the fundamental or $5 D$ parameters of the theory [18]. Remarkably, the correspondence between $\mathrm{AdS}_{5}$ and $4 D$ conformal field theories (CFT) [19] suggests that the scenario with warped extra dimension is dual to the idea of a composite Higgs in $4 D$ [20, 17].

It was realized that with SM fermions propagating in the bulk, we can also account for the hierarchy between quark and lepton masses and mixing angles (flavor hierarchy) [3, 4]. The basic idea is that light SM fermions - which are the zero-modes of $5 D$ fermions - can be localized near 
the Planck brane, resulting in a small overlap with the TeV-brane localized SM Higgs, while the top quark is localized near the $\mathrm{TeV}$ brane with a large overlap with the Higgs. Again, the crucial point is that such vastly different profiles can be realized with small variations in the $5 D$ mass parameters of fermions, i.e., without any large hierarchies in the parameters of the $5 D$ theory. Due to the different profiles of the SM fermions in the extra dimension, flavor changing neutral currents (FCNC) are generated by their non-universal couplings to gauge KK states. However, these contributions to the FCNC's are suppressed due to an analog of the Glashow-Iliopoulos-Maiani (GIM) mechanism of the SM, i.e. RS-GIM, which is "built-in" [4, 21, 22]. The point is that all KK modes (whether gauge, graviton or fermion) are localized near the $\mathrm{TeV}$ or IR brane (just like the Higgs) so that non-universalities in their couplings to SM fermions are of same size as couplings to the Higgs.

In spite of this RS-GIM suppression, it was shown recently [23] (see also [24, 25]), that the constraint on the KK mass scale from contributions of KK gluon to $\epsilon_{K}$ is quite stringent. In particular, for the model with the SM Higgs (strictly) localized on the TeV brane, the limit on the KK mass scale from $\epsilon_{K}$ is $\sim 10-40 \mathrm{TeV}$, depending on the size of the $5 D$ QCD gauge coupling. However, the phenomenology of the $\mathrm{TeV}$-scale KK modes and the SM Higgs is quite sensitive to the structure near the $\mathrm{TeV}$ brane (where these particles are localized). For example, the SM Higgs can be the lightest mode of a $5 D$ scalar (instead of being a strictly $\mathrm{TeV}$ brane-localized field), but with a profile which is still peaked near the TeV brane (such that the Planck-weak hierarchy is still addressed) i.e. a "bulk Higgs" [26]. Moreover, the warped geometry might deviate from pure AdS near the TeV brane which in fact could be replaced with a "soft wall" [27]. Similarly, in general, there are non-zero TeV brane-localized kinetic terms for the bulk fields [28]. Such variations of the minimal models are not likely to modify the constraint on KK mass scale from various precision tests by much more than $O(1)$ factors. However, even such modest changes can dramatically impact the LHC signals, especially the production cross-sections for the KK modes.

With the above motivation, the "two-site model" [29] was proposed as an economical description of this framework in order to capture the robust aspects of the phenomenology by effectively restricting to the SM fields and their first KK excitations. In reference [30], it was shown that a mass scale for the new particles as low as $\sim O(5) \mathrm{TeV}$ is consistent with the combination of constraints from $\epsilon_{K}$ and $\mathrm{BR}(b \rightarrow s \gamma)$, and it was suggested that models with a bulk Higgs can allow a similar KK scale. In addition, mechanisms exist to ameliorate such constraints in a parametric manner, for example through flavor symmetries [31, 24] or by lowering the UV-IR hierarchy [32], as opposed to simply relying on the $O(1)$ effects mentioned above.

Most of the studies of the KK gluon, graviton and $Z$ (and similarly our study of the KK $W$ here) focus on flavor-preserving fermionic decays (i.e., $t \bar{t}$ for neutral and $t \bar{b}$ for charged case), except for Ref. [33] which considers flavor-violating decays of the KK gluon. So, it is important to point out that the results of these studies apply to the warped extra dimensional framework independent of 
the specific mechanism used to suppress flavor violation (beyond that from the RS-GIM mechanism) since the profiles and hence the (flavor -preserving) couplings remain (roughly) the same in all these different models for suppressing flavor violation (except in Ref. [32] with UV-IR hierarchy being smaller than Planck-weak). For other studies of flavor physics, see Refs. [34, 35].

Finally, various custodial symmetries [36, 37] can be incorporated such that the constraints from the various (flavor-preserving) electroweak precision tests (EWPT) can be satisfied for a few $\mathrm{TeV}$ KK scale [36, 38]. The bottom line is that a few TeV mass scale for the KK gauge bosons can be consistent with both electroweak and flavor precision tests.

\subsection{Couplings}

Clearly, the light fermions have a small couplings to all KK's (including graviton) based simply on the overlaps of the corresponding profiles, while the top quark and Higgs have a large coupling to the KK's. Schematically, neglecting effects related to EWSB, we find the following ratio of RS1 to SM gauge couplings:

$$
\begin{aligned}
\frac{g_{\mathrm{RS}}^{q \bar{q} l l \bar{l} A^{(1)}}}{g_{\mathrm{SM}}} & \simeq-\xi^{-1} \approx-\frac{1}{5} \\
\frac{g_{\mathrm{RS}}^{Q^{3} \bar{Q}^{3} A^{(1)}}}{g_{\mathrm{SM}}}, \frac{g_{\mathrm{RS}}^{t_{R} \bar{t}_{R} A^{(1)}}}{g_{\mathrm{SM}}} & \simeq 1 \text { to } \xi(\approx 5) \\
\frac{g_{\mathrm{RS}}^{H H} A^{(1)}}{g_{\mathrm{SM}}} & \simeq \xi \approx 5 \quad\left(H=h, W_{L}, Z_{L}\right) \\
\frac{g_{\mathrm{RS}}^{A^{(0)} A^{(0)} A^{(1)}}}{g_{\mathrm{SM}}} & \sim 0
\end{aligned}
$$

Here $q=u, d, s, c, b_{R}, l=$ all leptons, $Q^{3}=(t, b)_{L}$, and $A^{(0)}\left(A^{(1)}\right)$ correspond to zero (first KK) states of the gauge fields. Also, $g_{\mathrm{RS}}^{x y z}, g_{\mathrm{SM}}$ stands for the RS1 and the three SM (i.e., $\left.4 D\right)$ gauge couplings respectively. Note that $H$ includes both the physical Higgs $(h)$ and unphysical Higgs, i.e., longitudinal $W / Z$ by the equivalence theorem (the derivative involved in this coupling is similar for RS1 and SM cases and hence is not shown for simplicity). Finally, the parameter $\xi$ is related to the Planck-weak hierarchy: $\xi \equiv \sqrt{k \pi r_{c}}$. EWSB induces mixing between EW KK states which we discuss in App. A

For completeness, we present the couplings of the KK graviton to the SM particles. These couplings involve derivatives (for the case of all SM particles), but (apart from a factor from the overlap of the profiles) it turns out that this energy-momentum dependence is compensated (or made dimensionless) by the $\bar{M}_{P} e^{-k \pi r_{c}} \sim \mathrm{TeV}$ scale, instead of the $\bar{M}_{P}$-suppressed coupling to the 
SM graviton. Again, schematically:

$$
\begin{aligned}
& g_{\mathrm{RS}}^{q \bar{q}, l \bar{l} G^{(1)}} \sim \frac{E}{\bar{M}_{P} e^{-k \pi r_{c}}} \times 4 D \text { Yukawa } \\
& g_{\mathrm{RS}}^{A^{(0)} A^{(0)} G^{(1)}} \sim \frac{1}{k \pi r_{c}} \frac{E^{2}}{\bar{M}_{P} e^{-k \pi r_{c}}} \\
& g_{\mathrm{RS}}^{Q^{3} \bar{Q}^{3} A^{(1)}}, g_{\mathrm{RS}}^{t_{R} \bar{t}_{R} G^{(1)}} \sim\left(\frac{1}{k \pi r_{c}} \text { to } 1\right) \frac{E}{\bar{M}_{P} e^{-k \pi r_{c}}} \\
& g_{\mathrm{RS}}^{H H G^{(1)}} \sim \frac{E^{2}}{\bar{M}_{P} e^{-k \pi r_{c}}}
\end{aligned}
$$

Here, $G^{(1)}$ is the KK graviton and the tensor structure of the couplings is not shown for simplicity.

Next, we briefly review studies of LHC signals in this scenario.

\subsection{LHC signals}

Based on these couplings, and the fact that precision electroweak and flavor constraints require the mass to be bigger than a few $\mathrm{TeV}$, we are faced with the following challenges in obtaining the EW KK gauge boson signals at the LHC from direct production of the KK modes, namely,

(i) Cross-section for production of these states is suppressed to begin with due to a small coupling to the protons' constituents, and due to the large mass;

(ii) Decays to "golden" channels (leptons, photons) are suppressed. Instead, the decays are dominated by top quark and Higgs (including longitudinal $W / Z$ );

Also, these resonances tend to be quite broad due to the enhanced couplings to top/Higgs.

In particular, the KK graviton, gluon and neutral electroweak gauge bosons all have sizable branching ratio (BR) to decay to top pairs. Moreover, due to the large mass (few TeV) of the KK particle, the top quarks produced in their decays are highly boosted, resulting in a high degree of collimation of the top quark's decay products. Hence it is a challenge to identify these top quarks. Nonetheless, using the techniques suggested in Refs. [8, 9] (see also Refs. [39] for related studies and [40, 41] for recent developments of the techniques for detecting highly boosted top quarks), discovery for $\mathrm{KK}$ gluon up to $\sim 4 \mathrm{TeV}$ mass might be possible. However, in the case of approximately degenerate gauge KK modes, it is still difficult to extract the signal from top pairs for electroweak neutral KK modes. The reason is that the top pair signal from these states is swamped by the decays of the KK gluon which has a (much) larger cross-section than that of the KK electroweak neutral gauge boson due to the QCD coupling and color factors, even though the SM $t \bar{t}$ background might be smaller than the electroweak neutral KK signal.

As mentioned above, couplings of KK's to longitudinal $W / Z$ are also enhanced similarly to top quarks (of course, only for KK graviton and electroweak - both neutral and charged - KK modes) so that decays to these modes also have a significant BR. Such final states are a priori 
cleaner than top quarks, in particular, since there is no "pollution" from QCD or KK gluon and there are decay channels with no jets in these cases. Hence such final states might be the discovery modes for electroweak (both charged and neutral) and graviton KK states. However, we still face some challenges in discovering the neutral electroweak (cf. charged case below) and graviton KK states even with the $W / Z$ final states as follows. The purely leptonic decay in the $W W$ channel has a small $\mathrm{BR}$ and moreover, the $W W$ invariant mass cannot be fully reconstructed due to the presence of missing momentum from two $\nu$ 's. Since it is difficult then to apply the mass window cut efficiently (i.e., without reducing signal) in order to isolate the events in the resonance region only, the SM background tends to be larger. It is true that the $Z Z \rightarrow 4 l$ final state can be fully reconstructed, but it has an even smaller BR to leptons than the $W W$ channel and is available only for the the graviton (it is absent for the neutral electroweak gauge KK).

On the other hand, the semileptonic decay of the $W W$ from the KK $Z$ and graviton has a larger BR. However, just as for the top quarks mentioned above, the hadronic decays of the highly boosted $W / Z$ pose a challenge for detection: the 2 jets from $W / Z$ tend to merge so that the QCD $W / Z+$ jet background (where a QCD jet fakes a hadronically decaying $W / Z$ ) becomes significant. Of course, this background is reducible so that with suitable discriminators between QCD and $W / Z$ jets such as jet mass, this channel can still be useful [13]. A similar argument applies to decays of KK $W$ to $W Z$.

In this paper, we study LHC signals from direct production of charged electroweak KK gauge bosons in the framework of a warped extra dimension. Apart from completing the study of spin-1 KK's, our motivation for this study is that these states possess some new features relative to KK neutral electroweak gauge boson and graviton, namely,

(i) The fully leptonic (and hence clean) decay mode of the $W Z$ channel can be fully reconstructed 1 due to the presence of only one $\nu$. Hence, it is expected that the signal to background ratio can be enhanced efficiently by a suitable cut on the $W Z$ invariant mass, namely, by simply requiring this mass to lie in the resonance region (unlike for the neutral case discussed above). Moreover, this final state for the $W Z$ has a larger BR than the $Z Z \rightarrow 4 l$ case for the $\mathrm{KK}$ graviton (although leptonic $\mathrm{BR}$ of $W Z$ is smaller than that of the fully leptonic decay of the $W W$ final state for the KK graviton and neutral electroweak gauge boson).

The issues with the semileptonic decay of $W Z$ will be similar to that in the neutral electroweak gauge boson case.

(ii) Decays to top + bottom final state of electroweak charged gauge bosons can be also reconstructed even for the leptonic decay mode of the top quark.2 The irreducible SM background

\footnotetext{
${ }^{1}$ assuming that the missing momentum from the neutrino combined with the lepton forms a $W$, or assuming that the neutrino 3-momentum is collinear with that of the lepton due to the large boost of the $W$ in the lab frame.

${ }^{2}$ imposing the on-shell conditions for $M_{W}$ and $m_{t}$.
} 
from the electroweak process (single top production) can be shown to be smaller than the signal inside the resonance region. Compared to the case of the neutral electroweak KK's where the decays to top pairs have an irreducible SM background from QCD processes, the electroweak background for the charged case is smaller (and the signal cross-section for the neutral case is roughly similar to the charged case). Moreover, if the KK gauge bosons are degenerate, then an even larger background from the KK gluon decays to top pairs completely swamps the signal from the electroweak neutral KK boson.

However, even for the charged case, QCD top pairs (from KK gluon or the SM) can be a significant background if one top quark fakes a bottom quark due to collimation of its decay products. Of course, techniques to distinguish a highly boosted top from a bottom can suppress this background, i.e., it is a reducible one.

\subsection{Overview of the charged electroweak gauge boson sector}

We present the full details on the model we work with along with a derivation of all the $W^{\prime}$ couplings in Appendix (App) $\mathrm{A}$ and $\mathrm{B}$. Here, we summarize some of the salient features of the various cases that we study in detail in the next two sections. First of all, due to the extended EW gauge symmetry in the bulk, i.e., $S U(2)_{L} \times S U(2)_{R} \times U(1)_{X}$ which is motivated by suppressing contributions to the $T$ parameter, we see that there are two charged KK towers (one from each $S U(2)$ group), before EWSB. We will restrict to the 1st mode of each tower, denoting these states by $W_{L_{1}, R_{1}}$, respectively. EWSB will mix these states and the resulting mass eigenstates will be denoted by $\tilde{W}_{L_{1}, R_{1}}$, or to reduce clutter, simply as $W_{L, R}^{\prime}$.

As explained above, these charged EW gauge bosons will decay mostly into Higgs, including (longitudinal) $W / Z$ and to top-bottom final states. In the appendix, we define two cases for the top-bottom sector (corresponding to different representations for the top-bottom sector under the $S U(2)_{L} \times S U(2)_{R}$ group) that we will consider in this work. Here we summarize the main features of the two cases (details are given in the appendix)

Case (i): $t_{R}$ has close-to-flat profile and $(t, b)_{L}$ has a profile localized very close to the $\mathrm{TeV}$ brane in the bulk

Case (ii): vice versa, i.e., $(t, b)_{L}$ has close-to-flat profile and $t_{R}$ has profile localized very close to the TeV brane in the bulk

Roughly speaking, flavor precision tests tend to (strongly) prefer case (ii), whereas EW precision tests have a (milder) preference for case (i).

Since, as shown in the appendix, the representations under $S U(2)_{L} \times S U(2)_{R} \times U(1)_{X}$ for these two cases are less than minimal, there are various "exotic" fermion fields included in these representations (cf Eqs. (22) and (23)) in addition to the SM fermions. These exotic fermions can 


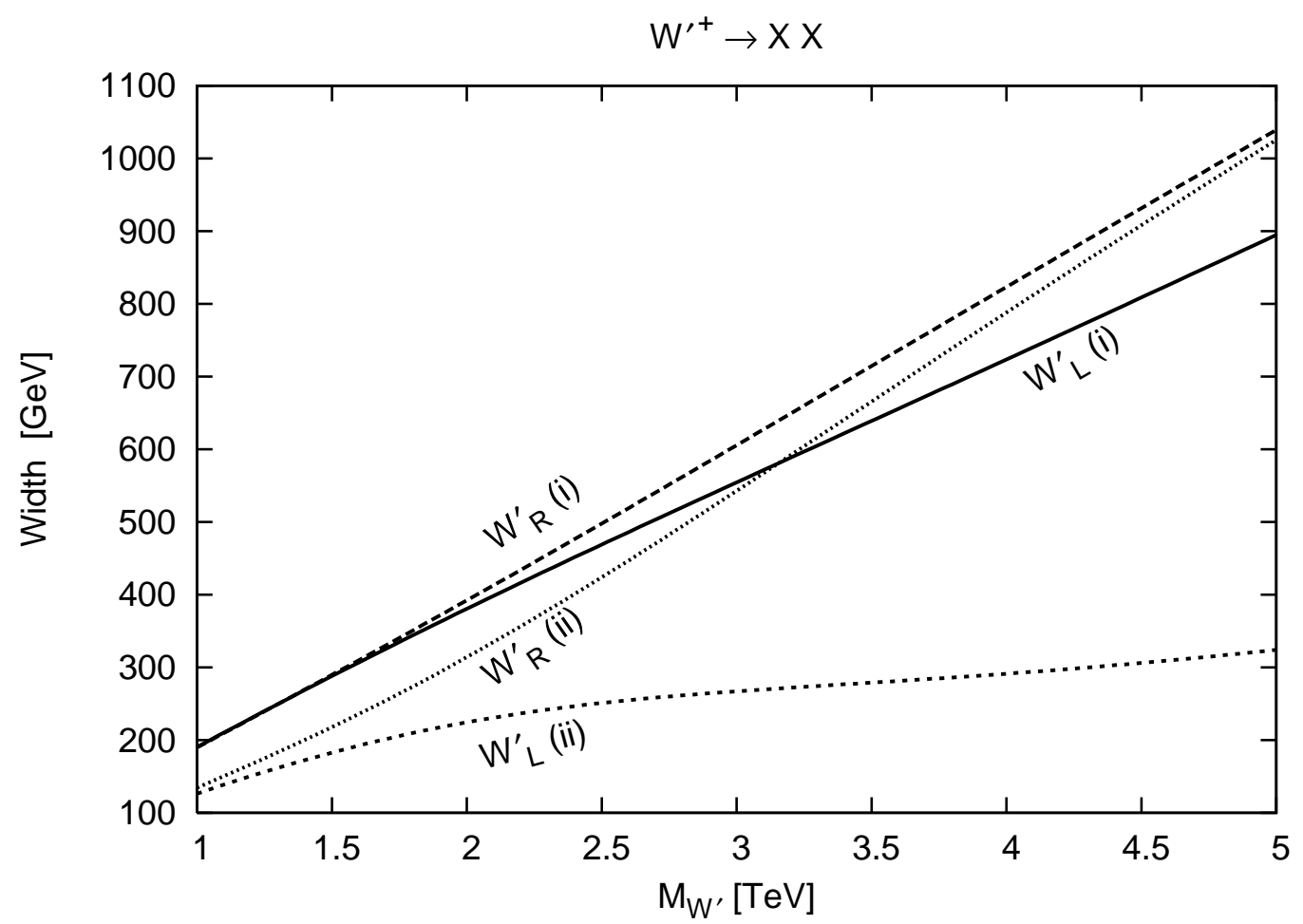

Figure 1: The total widths of $W_{L}^{\prime}$ and $W_{R}^{\prime}$ as a function of their masses for cases (i) and (ii).

be looked for at the LHC but we will not consider them here; instead we will restrict ourselves to SM final states. However, in order to obtain realistic values, we will include these exotic decay channels in computing the BR's.

\section{$3 \quad W^{\prime}$ decays}

The overlap integrals that dictate the $W^{\prime}$ couplings to fermions in the four-dimensional effective theory are presented in Table 10. We then derive the $W^{\prime}$ couplings to gauge bosons in the rest of App. B. Armed with these couplings, we are ready to embark on the phenomenology of charged EW gauge bosons in this framework. We have incorporated the $W^{\prime}$ couplings shown in App. B into the Monte Carlo program CalcHEP [42], using which we present the results below. We will refer to the mass eigenstates $\tilde{W}_{L_{1}}$ and $\tilde{W}_{R_{1}}$ as just $W_{L}^{\prime}$ and $W_{R}^{\prime}$ respectively, i.e., we will always work with mass eigenstates while studying the phenomenology. In Fig. 1 we show the total widths of the $W_{L}^{\prime}$ and $W_{R}^{\prime}$ into 2-body final states as a function of their masses for cases (i) and (ii). The total width increases monotonically with $M_{W^{\prime}}$ as expected, and is roughly about $20 \%$ or less of its mass, with it being appreciably smaller for the $W_{L}^{\prime}$ in case (ii). This implies a typically weakly interacting particle and a prompt decay, although the width can still be rather large for a high mass. A distinct feature is that the total width of $W_{L}^{\prime}$ in case (ii) is much smaller than all the others for 

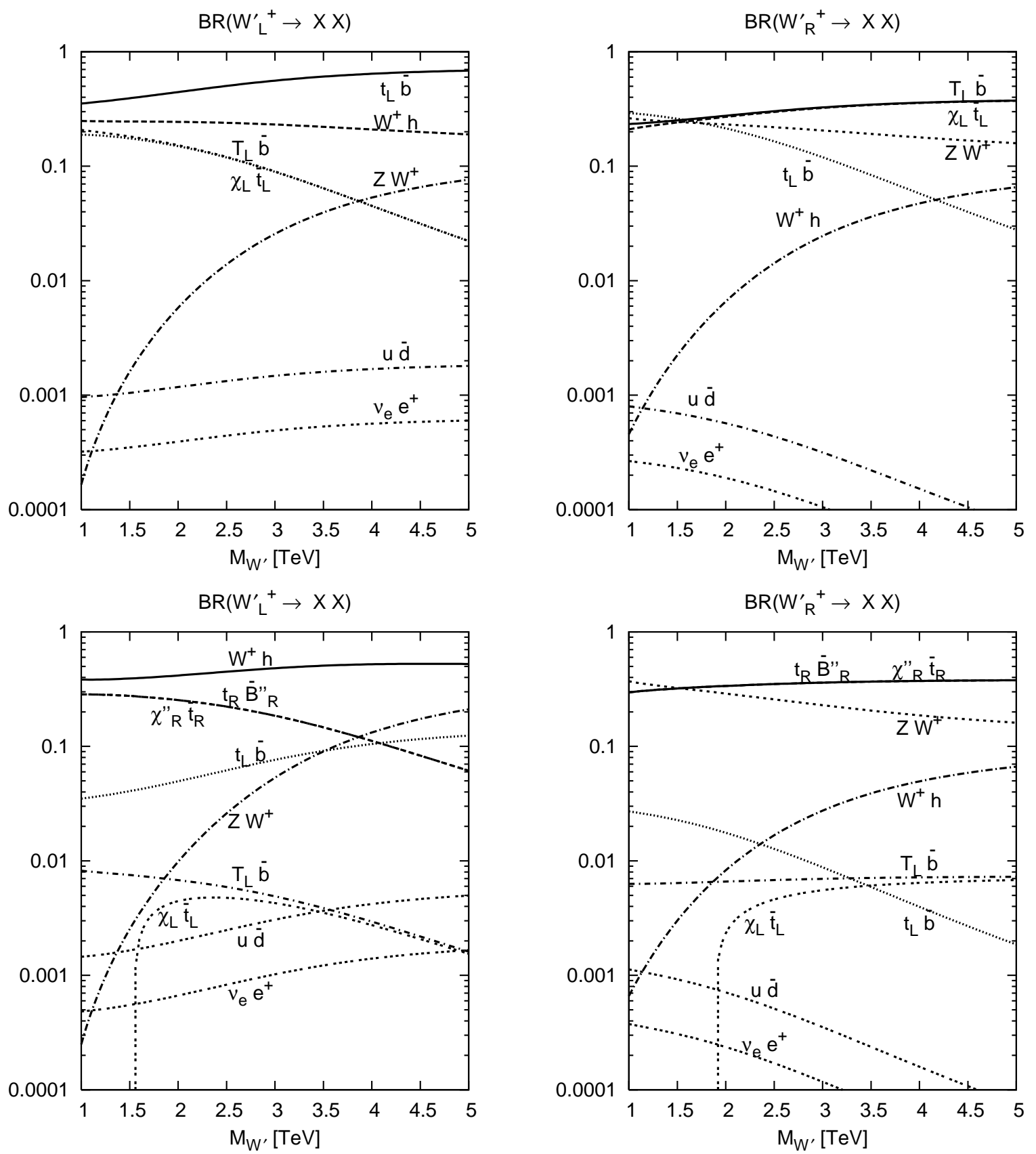

Figure 2: The branching fractions of $W_{L}^{\prime}$ (left) and $W_{R}^{\prime}$ (right) as a function of their masses for case (i) (top panels), and case (ii) (bottom panels). In the bottom panel for case (ii), note that the curves for $t_{R} \bar{B}_{R}^{\prime \prime}$ and $\chi_{R}^{\prime \prime} \bar{t}_{R}$ are on top of each other and cannot be individually differentiated. 
large $M_{W^{\prime}}$. This is due to the fact that in case (ii) there is no direct coupling of the $W_{L_{1}}$ to a third generation fermion whose wavefunction is peaked toward the $\mathrm{TeV}$ brane, unlike in the other cases. This is a direct result of the $S U(2)_{L} \otimes S U(2)_{R}$ quantum numbers of the third generation fermions (cf Eqs. (22) and (23) ). Note however that it can still be coupled to a TeV-brane peaked state via $W_{L_{1}} \leftrightarrow W_{R_{1}}$ mixing, but this would be suppressed by this mixing angle which is small for large $M_{W^{\prime}}$.

In Fig. 2 we show the branching fractions (BR) of the mass eigenstates $W_{L}^{\prime}$ (left panel) and $W_{R}^{\prime}$ (right panel) into various 2-body final states for cases (i) (top panels) and (ii) (bottom panels). The largest branching fraction is to fermions peaked toward the $\mathrm{TeV}$ brane, which in case (i) is to $Q_{L}^{3}$ modes, while for $W_{R}^{\prime}$ in case (ii) it is to the triplet containing $t_{R}$. In contrast, for the $W_{L}^{\prime}$ in case (ii) the largest $\mathrm{BR}$ is to $W h$ since there is no direct coupling to a third generation fermion with a $\mathrm{TeV}$ brane peaked profile. For the $W_{R}^{\prime}$ in case (ii), the $Z W$ final state is also available with a sizable BR. In case (ii) the $\chi_{L} \overline{t_{L}}$ is available only for $M_{W^{\prime}}>M_{\chi_{L}}+M_{t}$, and therefore exhibits a threshold behavior.

In this work we do not study the exotic particles in the final states, and we will focus on the $t \bar{b}, Z W$ and $W h$ final states in the rest of the paper. The BR into $\ell \nu$ final state is tiny, but due to its uniqueness and for completeness we will briefly comment on this mode also. We will perform a detailed study of these final states considering their various decay modes and obtain the LHC reach.

\section{Charged Gauge Boson Signals at the LHC}

In this section we consider the production of the charged KK gauge bosons (generically denoted by $W^{\prime}$ ) and its decay into various SM final states at the LHC. We first present in Fig. 3 the total production cross-section for the $W^{\prime}$ at the LHC versus its mass $M_{W^{\prime}}$ via the Drell-Yan (DY) process. We see that the cross-section can be $100-1 \mathrm{fb}$ for $M_{W^{\prime}}=2-4 \mathrm{TeV}$. The $W_{R}^{\prime}$ coupling to light quarks is suppressed by the $W_{L_{1}} \leftrightarrow W_{R_{1}}$ mixing angle (cf Eq. 24) and the rate is therefore smaller by a factor of $2-10$ in the mass range mentioned above. In the following analyses, we coherently sum the $W_{L}^{\prime}$ and $W_{R}^{\prime}$ (the mass eigenstates) contributions. There are other possible mechanisms for $W^{\prime}$ production. One may consider the gauge boson fusion $W Z \rightarrow W^{\prime}$. However, the gauge-boson fusion channel, as first explored in [13, was found to be subleading. As mentioned earlier, we adopt the Monte Carlo package CalcHEP [42] to obtain the numerical results in this section. We use the CTEQ6M for parton distribution functions [43]. 


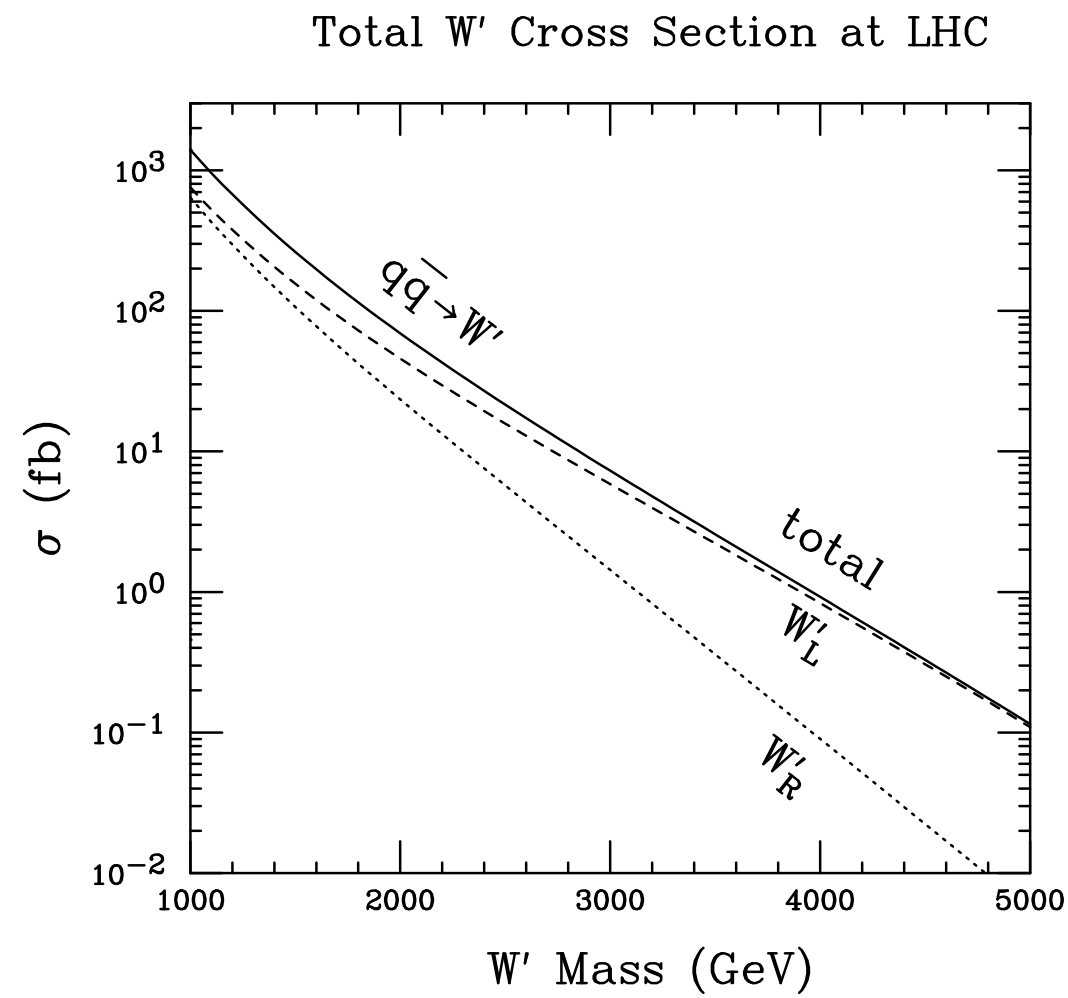

Figure 3: Total production cross-section for $W^{\prime}$ versus its mass at the LHC.

\section{$4.1 t \bar{b}$ final state}

We first consider the production and decay channel

$$
p p \rightarrow W^{\prime+} \rightarrow t \bar{b} \quad \text { with } \quad t \rightarrow b \ell \bar{\nu} \quad(\ell=e, \mu)
$$

where the leptonic decay modes of the top have been specified for the purpose of event triggering and identification. The most distinctive feature of this signal is the large invariant mass of the $t b$ system near $M_{W^{\prime}}$. Although the missing neutrino makes the event reconstruction less trivial, one should be able to reconstruct the neutrino momentum fairly effectively by demanding the two mass relations $M_{W}^{2}=\left(p_{l}+p_{\nu}\right)^{2}, m_{t}^{2}=\left(p_{b}+p_{l}+p_{\nu}\right)^{2}$. We thus assume that the signal events are fully reconstructible.

We select events with the basic acceptance cuts

$$
\left|y_{W, b, \bar{b}}\right|<3 ; \quad p_{T W, b, \bar{b}}>50 \mathrm{GeV}
$$

where the $y$ 's are the rapidities. For the signal events from a heavy $W^{\prime}$ decay, further tightened cuts can help for the background suppression. We thus impose the cuts on the top and $b$ quarks

$$
p_{T, b}>200 \mathrm{GeV}, \quad\left|y_{t, b}\right|<3 .
$$



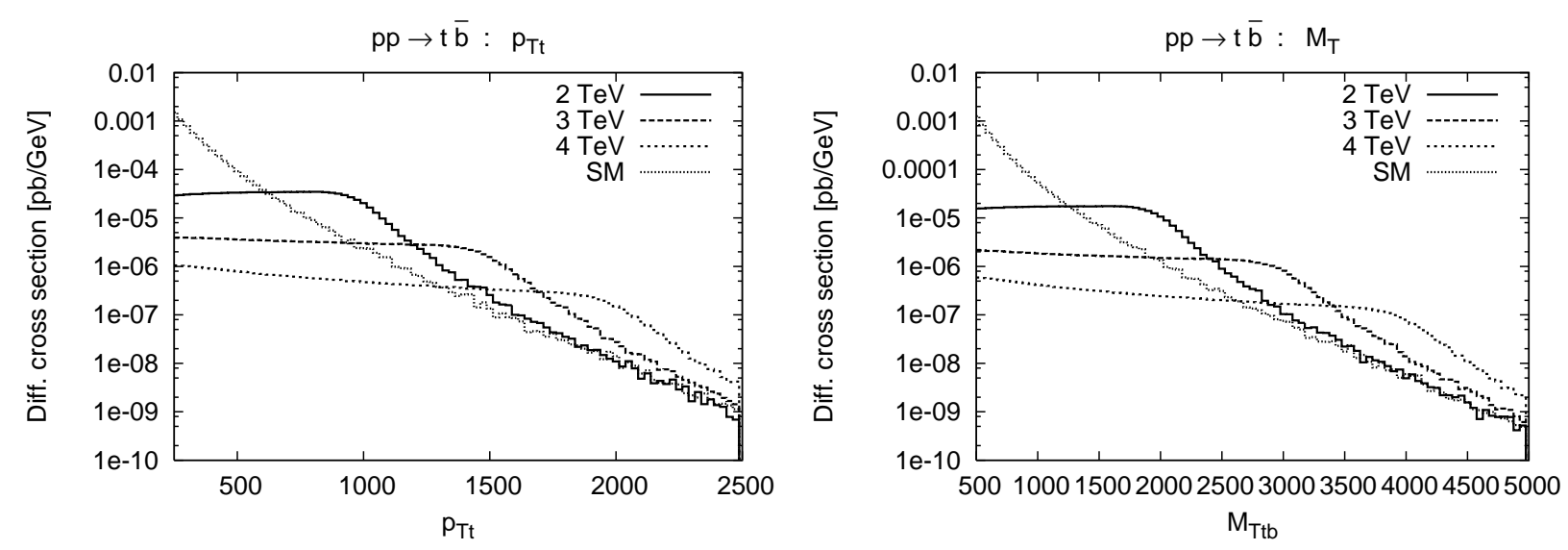

Figure 4: The (a) $p_{T t}$, and (b) $M_{T t b}$ differential distributions of the process $p p \rightarrow W^{\prime+} \rightarrow t \bar{b}$ for $M_{W^{\prime}}=2,3$ and $4 \mathrm{TeV}$ for case (i). These are after the cuts in Eq. (5). Also shown are the SM single top background distributions.

In Fig. 4, we present the differential distributions for the $t \bar{b}$ final state with a variety of values of $M_{W^{\prime}}=2,3$ and $4 \mathrm{TeV}$, for (a) the transverse momentum distribution, and (b) the transverse mass distribution. Also shown on the figures is the dominant source of irreducible background, the SM single top production $p p \rightarrow W^{+} \rightarrow t \bar{b}$, seen as the continuum curves. We see the very promising prospects for observing the signal with suitably chosen cuts.

With the decay of the top (into $W b$ ), the SM $W b \bar{b}$ will be an additional source of background. Since this background largely populates the low mass threshold region, we thus form the following cluster transverse mass to help distinguish the signal from background

$$
\begin{aligned}
M_{T W b} & =\left(\sqrt{p_{T W}^{2}+m_{W}^{2}}+p_{T b}\right)^{2}-\left|\mathbf{p}_{T_{W}}+\mathbf{p}_{T_{b}}\right|^{2}, \\
M_{T W b \bar{b}} & =p_{T b}+p_{T \bar{b}}+\sqrt{p_{T_{W}}^{2}+m_{W}^{2}} .
\end{aligned}
$$

We show the representative kinematical distributions for the $W b \bar{b}$ final states in Fig. 5 , with a $M_{W^{\prime}}=2 \mathrm{TeV}$ signal (solid curves) for illustration. The SM backgrounds of $t \bar{b}$ (dashed) and $W b \bar{b}$ (dotted) are also shown for comparison. Figure 5(a) presents the transverse momentum distribution for the $W$. In Fig. 5 (b), we show the distribution of $M_{T W b}$, the cluster transverse mass of the $W$ with the nearer of the two b-jets. The top-quark mass reconstruction is visible for those events with a real top in the final state. We show in Fig. 5(c) the distribution of $\cos _{T} \theta_{W b}$, the $\operatorname{cosine}$ of the angle in the transverse plane between the $W$ and the nearer of the two b-jets. Due to the large boost of the top quark from the $W^{\prime}$ decay for the signal, the opening angle obviously is rather small as seen by the solid curve. In Fig. 5(d), we show the distribution of the full transverse mass of the $W b \bar{b}$ system. It is encouraging to see a possible separation of the signal from the backgrounds.

Since there is only one missing neutrino, the kinematical variables can be fully reconstructed 

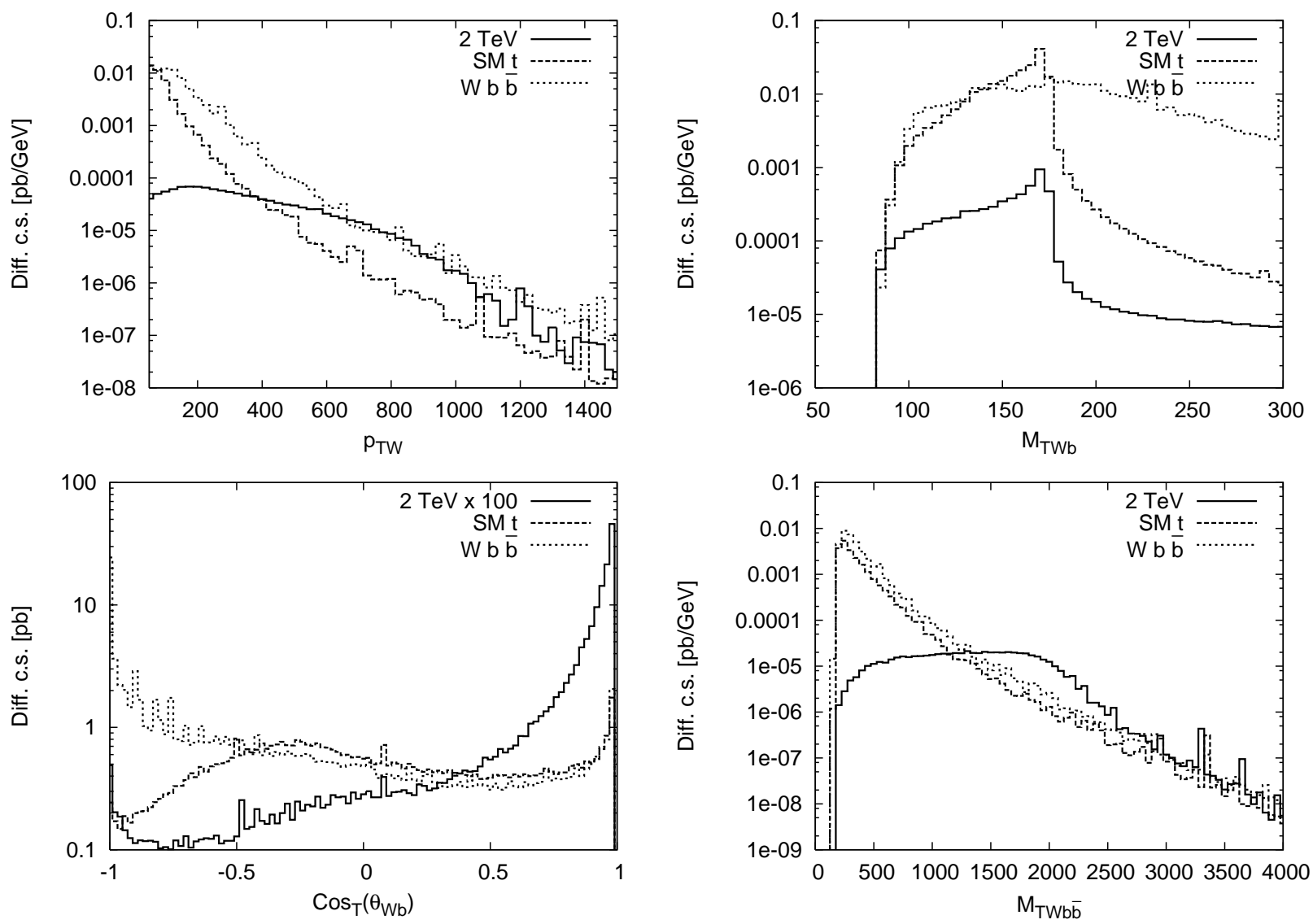

Figure 5: The (a) $p_{T W}$, (b) $M_{T W b}$, (c) $\cos _{T} \theta_{W b}$, and (d) $M_{T W b \bar{b}}$ differential distributions of the process $p p \rightarrow W^{+} b \bar{b}$ for $M_{W^{\prime}}=2 \mathrm{TeV}$, for case (i). Also shown are the irreducible backgrounds, the SM single top $t \bar{b}$ and $W b \bar{b}$. 
in the event as discussed earlier by demanding the mass reconstruction of $M_{W}, m_{t}$. Alternatively, since the $W$ is produced with a large boost, the neutrino will be considerably collimated with the charged-lepton. If one makes the assumption $3 \vec{p}_{\nu} \approx \kappa \vec{p}_{\ell}$, the neutrino 4-momentum can be approximately determined and the full $M_{W b \bar{b}}$ can be formed. We find that doing so gives a narrower signal invariant-mass peak, but also raises the background in the region of interest, resulting in a marginal improvement in significance; we therefore do not pursue either of these ideas here.

The distributions in Fig. 5 motivate us to consider the following cuts: $M_{T W b}$ cut: $100<M_{T W b}<190 \mathrm{GeV}$, since we expect for the signal this should reconstruct to the parent $m_{\text {top }}$, as can be seen in Fig. [5). Notice that since for single top this also reconstructs to $M_{\text {top }}$, this variable does not discriminate between the signal and this source of background.

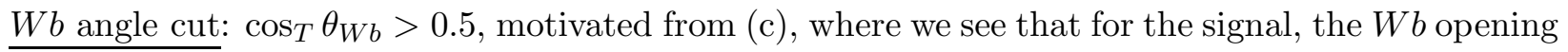
angle is fairly small owing to the large boost of the parent top.

$2 b$-tags: We demand that there be two tagged b's in the event. We adopt a $b$-tagging efficiency $\eta_{b}=0.4$ [44]. With $b$-tagging parameters optimized for low $p_{T b}$ the light-quark rejection ratio (for $j=u, d, s, g)$ is roughly $R_{j}=20$ [44], where $1 / R_{j}$ is the probability of mistaking a light-jet for a b-jet. We believe this is likely to be improved with tagging techniques optimized for high $p_{T b}$, and since our light-quark jet background is significant, we anticipate such improvements and use $R_{j}=40$. We use a charm quark rejection factor $R_{c}=5$.

$\underline{M_{T W b B} \text { cut: }} 1500<M_{T W b \bar{b}}<2500 \mathrm{GeV}$ (for $M_{W^{\prime}}=2 \mathrm{TeV}$ ), and, $2400<M_{T W b \bar{b}}<3600 \mathrm{GeV}$ (for $M_{W^{\prime}}=3 \mathrm{TeV}$ ), which is motivated by the resonant feature of the signal as seen clearly in (d), and results in the background being very effectively suppressed after this cut.

Jet-mass cut: $t \bar{t}$ production can become a source of background since a top can fake a $b$-jet, for instance when the hadronic decay products of a boosted top are sufficiently collimated that it can be confused for a $b$-jet. The two main sources of a top pair are the SM QCD production, and the KK-gluon production which dominantly decays to this channel. Both can be significantly larger than the signal, and the latter is especially problematic since it is resonant in the same invariant mass region as the signal. However, the jet-mass variable can be used to discriminate between a $b$-jet and a boosted hadronic top, with the distributions expected to peak at $m_{b}$ and $m_{t}$ respectively. In order to obtain a rough estimate of the separation achievable, we have used Pythia v6.411 [45] to shower a bottom and a hadronically decayed top, followed by smearing the daughter particles energy by $80 \% / \sqrt{E}$, and $\eta$ and $\phi$ by 0.05 to mimic the finite resolutions of the detector 4 In general, a larger cone-size will include more of the radiation and results in a narrower distribution for $t$-jets but at the expense of moving the $b$-jet peak to larger values. Also, since a $b$-jet is expected

\footnotetext{
${ }^{3}$ Practically, $p_{T}(\nu)=\not_{T}, p_{L}(\nu)=p_{L}(\ell) \times \frac{\not \phi_{T}}{p_{T}(\ell)}$.

${ }^{4}$ We are grateful to Frank Paige for many discussions on jet-mass issues. This variable was also explored in Ref. 13 for the jet-mass of the $W$. Related issues, including using sub-structure of jets to reduce QCD backgrounds, have also been discussed in Refs. [9, 40, 41, 46].
} 

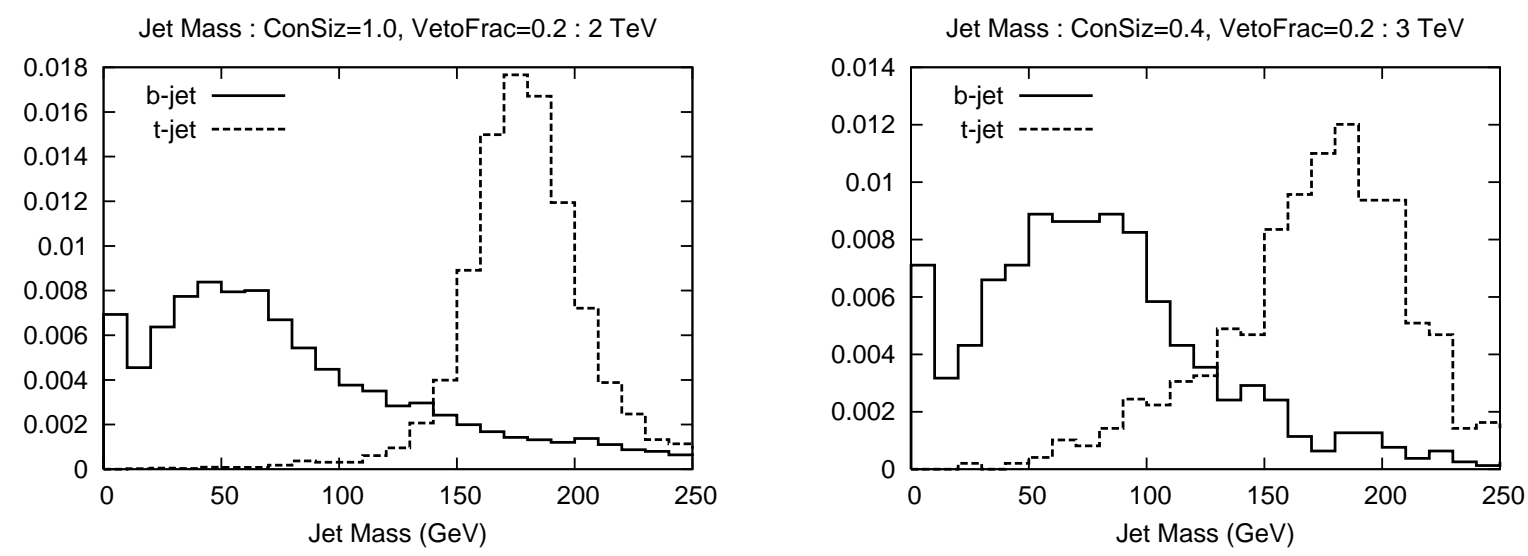

Figure 6: The jet-mass distributions for top and bottom jets. These are after basic, $W b$ and invariant-mass cuts. On the left is for $2 \mathrm{TeV}$ with cone-size 1.0, and, on the right for $3 \mathrm{TeV}$ with cone-size 0.4. Both are with veto-fraction of 0.2 .

to be more collimated than a $t$-jet, we also demand that $80 \%$ of the $p_{T}$ be contained within the cone (veto-fraction of 0.2 ). We show in Fig. 6 the resulting jet-mass distributions for the $2 \mathrm{TeV}$ (left) and $3 \mathrm{TeV}$ (right) cases. For the $2 \mathrm{TeV}$ case, we find a cone-size of 1.0 to result in adequate separation, and a jet-mass cut $M_{j}<75 \mathrm{GeV}$ with a veto-fraction of 0.2 retains $46 \%$ of b-jets and $0.38 \%$ of $t$-jets. For the $3 \mathrm{TeV}$ case, the decay products are more collimated and we therefore pick a smaller cone-size, namely 0.4 , and again with a veto-fraction of 0.2 . We find that, with the cut $M_{j}<100 \mathrm{GeV}$ (larger than the previous case in order to keep more of the already small signal events), $57 \%$ of $b$-jets and $2.5 \%$ of $t$-jets are retained.

In Table 1 we present the signal and background cross-sections (in fb) for the process $p p \rightarrow$ $W b \bar{b} \rightarrow \ell \nu b \bar{b}$ for case (i). We include in the signal both $W^{\prime+}$ and $W^{\prime-}$, and we find that the latter cross-section is about a third of the former, stemming from the difference in the PDF's of more $u$ quarks than $d$ quarks in a proton. We count for both $\ell=e, \mu$. Due to the large boost of the parent top, the lepton may not have a large isolation with respect to the b-jet, but we will assume that this will not result in too large a loss of efficiency. Ways to deal with this has been discussed in Refs. [8, 40]. The entry labeled as "SM top", in addition to the SM $W^{ \pm}$-exchange single-top process, also includes the $W$-glue fusion process containing an extra jet that we use to veto events with $p_{T_{j}}>20 \mathrm{GeV}$ in the central region. In the last two columns we show the significance without and with $t \bar{t}$ as a source of background, with the significance including the latter shown in parenthesis. The $G^{(1)}$ (KK gluon) is taken to be degenerate with $W^{\prime}$. From the table we see that we need $\mathcal{L}=100 \mathrm{fb}^{-1}\left(\mathcal{L}=300 \mathrm{fb}^{-1}\right)$ for a $2 \mathrm{TeV}(3 \mathrm{TeV}) W^{\prime}$, where we have estimated the signal significance by $S / \sqrt{B}$ in Gaussian distribution for large event sample, but by Confidence Level (CL) in Poisson statistics for small even sample. Although the $S / B$ is good for heavier masses, the signal will still be limited by statistics. 
Table 1: The cross-sections (in fb) for the signal process $p p \rightarrow W^{\prime} \rightarrow t b \rightarrow W \bar{b} b \rightarrow \ell \nu \bar{b} b$ for case (i), and SM background, with the cuts applied successively. Cross-sections are shown for $M_{W^{\prime}}=2$ and $3 \mathrm{TeV}$, with the number of events and significance for $\mathcal{L}=100$ and $300 \mathrm{fb}^{-1}$, respectively. In the last two columns we show the significance without and with $t \bar{t}$ as a source of background, with the significance including the latter shown in parenthesis.

\begin{tabular}{|c|c|c|c|c|c|c|c|c|}
\hline $2 \mathrm{TeV}, 100 \mathrm{fb}^{-1}$ & Basic & $W b$ cuts & b-tag & $M_{T W b b}$ & $M_{j}$ & $\#$ Evt & $S / B$ & $S / \sqrt{B}$ \\
\hline \hline Case (i) & 8.9 & 7 & 1.1 & 0.44 & 0.2 & 20 & $2.5(1.4)$ & $7(5.3)$ \\
\hline SM top & 1400 & 370 & 60 & 0.09 & 0.04 & 4 & & \\
\hline SM $W b b$ & 520 & 66 & 11 & $9 \times 10^{-3}$ & $4 \times 10^{-3}$ & 0.4 & & \\
\hline SM $W b j$ & $9 \times 10^{3}$ & $2 \times 10^{3}$ & 20 & 0.04 & 0.02 & 2 & & \\
\hline SM $W c j$ & $4 \times 10^{3}$ & 700 & 4 & $10^{-3}$ & $0.5 \times 10^{-3}$ & 0.05 & & \\
\hline SM $W j j$ & $2 \times 10^{5}$ & $2 \times 10^{4}$ & 13 & 0.03 & 0.01 & 1 & & \\
\hline \hline SM $t \bar{t}$ & $4 \times 10^{4}$ & $10^{4}$ & $2 \times 10^{3}$ & 4.5 & 0.02 & 2 & & \\
\hline$G^{(1)} t \bar{t}(\mathrm{i})$ & 250 & 190 & 30 & 10 & 0.04 & 4 & & \\
\hline
\end{tabular}

\begin{tabular}{|c|c|c|c|c|c|c|c|c|}
\hline $\begin{array}{c}3 \mathrm{TeV} \\
300 \mathrm{fb}^{-1}\end{array}$ & Basic & $W b$ cuts & b-tag & $M_{T W b b}$ & $M_{j}$ & $\#$ Evt & $S / B$ & $\begin{array}{c}\text { CL } \\
\text { Poisson }\end{array}$ \\
\hline \hline Case (i) & 1.5 & 1.1 & 0.18 & 0.04 & 0.02 & 7 & $5.8(0.9)$ & $0.995(0.95)$ \\
\hline SM top & 1400 & 370 & 60 & $4 \times 10^{-3}$ & $2 \times 10^{-3}$ & 0.6 & & \\
\hline SM $W b b$ & 520 & 66 & 11 & $4 \times 10^{-4}$ & $2.3 \times 10^{-4}$ & 0.07 & & \\
\hline SM $W b j$ & $9 \times 10^{3}$ & $2 \times 10^{3}$ & 20 & $10^{-3}$ & $0.5 \times 10^{-3}$ & 0.2 & & \\
\hline SM $W c j$ & $4 \times 10^{3}$ & 700 & 4 & $10^{-4}$ & $0.5 \times 10^{-4}$ & 0.02 & & \\
\hline SM $W j j$ & $2 \times 10^{5}$ & $2 \times 10^{4}$ & 13 & $2 \times 10^{-3}$ & $10^{-3}$ & 0.3 & & \\
\hline \hline SM $t \bar{t}$ & $4 \times 10^{4}$ & $10^{4}$ & $2 \times 10^{3}$ & 0.21 & $5.3 \times 10^{-3}$ & 1.6 & & \\
\hline$G^{(1)} t \bar{t}(\mathrm{i})$ & 32 & 24 & 4 & 0.64 & 0.02 & 5 & & \\
\hline
\end{tabular}

Table 2: The cross-sections (in fb) for the signal process $p p \rightarrow W^{\prime} \rightarrow t b \rightarrow W \bar{b} b \rightarrow \ell \nu \bar{b} b$ for case (ii), and SM background, with the cuts applied successively. Cross-sections are shown for $M_{W^{\prime}}=2 \mathrm{TeV}$, with the number of events and significance for $\mathcal{L}=1000 \mathrm{fb}^{-1}$. In the last two columns we show the significance without and with $t \bar{t}$ as a source of background, with the significance including the latter shown in parenthesis.

\begin{tabular}{|c|c|c|c|c|c|c|c|c|}
\hline $2 \mathrm{TeV}$ & Basic & $W b$ cuts & b-tag & $M_{T W b b}$ & $M_{j}$ & $\#$ Evt & $S / B$ & $S / \sqrt{B}$ \\
\hline \hline Case (ii) & 0.75 & 0.6 & 0.1 & 0.05 & 0.03 & 30 & $0.38(0.2)$ & $3.4(2.5)$ \\
\hline SM top & 1400 & 370 & 60 & 0.09 & 0.04 & 40 & & \\
\hline SM $W j_{1} j_{2}$ & $2.1 \times 10^{5}$ & $2.2 \times 10^{4}$ & 48 & 0.08 & 0.04 & 40 & & \\
\hline \hline SM $t \bar{t}$ & $4 \times 10^{4}$ & $10^{4}$ & $2 \times 10^{3}$ & 4.5 & 0.02 & 20 & & \\
\hline$G^{(1)} t \bar{t}(\mathrm{ii})$ & 210 & 180 & 29 & 13 & 0.05 & 50 & & \\
\hline
\end{tabular}


In Table 2 we present the signal and background cross-sections (in fb) for the process $p p \rightarrow$ $W b \bar{b} \rightarrow \ell \nu b \bar{b}$ for case (ii). Rather than repeating, in Table 2 we have combined, in SM $W j_{1} j_{2}$, the SM $W b \bar{b}, W b j, W c j$ and $W j j$ channels shown separately in Table 1. Compared to case (i), as expected, the cross-section is lower in case (ii) since the $t_{L}, b_{L}$ profiles are not peaked near the $\mathrm{TeV}$ brane (and hence do not have as large coupling to $W^{\prime}$ ) as in the former case, but rather it is the $t_{R}$ which either does not couple to $W^{\prime}$ if it is an $S U(2)_{R}$ singlet, or would couple to it only associated with an exotic fermion if it is a triplet. We find that we need a much higher luminosity,

namely, $1000 \mathrm{fb}^{-1}$ for a $2 \mathrm{TeV} W^{\prime}$. In this case, we expect the other channels (to be discussed in the following) to have better reach.

\section{2 $Z W$ final state}

As for the process

$$
p p \rightarrow W^{\prime} \rightarrow Z W
$$

we consider the gauge boson decay modes separately. In order to effectively reconstruct the final state, we do not pursue the missing decay channel $Z \rightarrow \nu \nu$. Although simultaneous hadronic decays of $Z$ and $W$ have the largest branching fraction, the multiple jet background from QCD would be overwhelming. We therefore do not pursue this mode in our study.

Since there is at most one missing neutrino in the final state, we can reconstruct the event if one makes the assumption $\vec{p}_{\nu} \approx \kappa \vec{p}_{e}$ (see the discussions in the last section). On the other hand, it is more straightforward to construct the events in the transverse plane. For illustration, we form the following kinematic variables:

$$
\begin{aligned}
M_{\text {eff } Z W} & =p_{T Z}+p_{T W} \\
M_{T Z W} & =\sqrt{p_{T Z}^{2}+M_{Z}^{2}}+\sqrt{p_{T W}^{2}+M_{W}^{2}}
\end{aligned}
$$

In Fig. 7 we show the $M_{e f f}{ }_{W}$ and $M_{T Z W}$ differential distributions for the process $p p \rightarrow Z W^{+}$for the $W^{\prime}$ signal for cases (i) and (ii), and the irreducible SM $W Z$ background.

\subsubsection{Fully leptonic channel}

In the fully leptonic final state we consider the process $p p \rightarrow W^{\prime} \rightarrow Z W$ followed by $Z \rightarrow \ell \ell$ and $W \rightarrow \ell \nu$. We take into account the SM $Z W$ going into the same final state as the main source of (irreducible) background. We select events with the basic cuts

$$
p_{T \ell}>50 \mathrm{GeV} ; \quad p_{T m i s s}>50 \mathrm{GeV} ; \quad\left|\eta_{\ell}\right|<3 .
$$

In addition to the basic cuts, we apply the following cuts sequentially in order to optimally improve signal observation from the background 

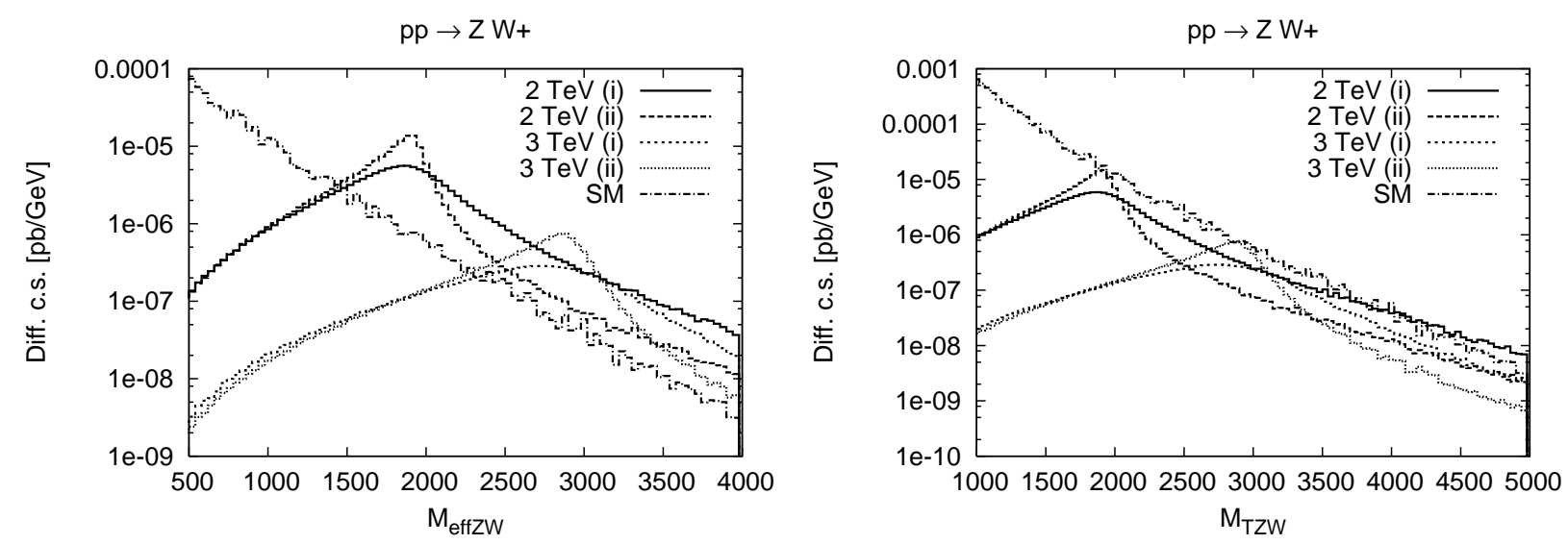

Figure 7: The $M_{\text {eff } Z W}$ (left) and $M_{T Z W}$ (right) distributions for signal and SM background for the process $p p \rightarrow Z W^{+}$after the cuts: $p_{T Z, W}>100 \mathrm{GeV}$ and $\left|y_{Z, W}\right|<3$. Both case (i) and case (ii) are shown.

Table 3: The cross-sections (in fb) for the signal process $p p \rightarrow W^{\prime} \rightarrow Z W \rightarrow \ell \ell \ell \nu$ for case (i) and case (ii), and SM background, with the cuts applied successively. We show cross-sections for $M_{W^{\prime}}=2$ and $3 \mathrm{TeV}$, and the number of events and significance with the luminosity $\mathcal{L}$ (in $\mathrm{fb}^{-1}$ ) as shown for each case.

\begin{tabular}{|c|c|c|c||c|c|c|c|}
\hline $2 \mathrm{TeV}$ & Basic & $M_{\text {eff }}$ & $M_{T}$ & $\mathcal{L}$ & \# Evts & $S / B$ & CL \\
\hline \hline Case (i) & 0.13 & 0.13 & 0.1 & 100 & 10 & 5 & 0.9995 \\
\hline Case (ii) & 0.17 & 0.16 & 0.13 & 100 & 13 & 6.5 & $>0.9995$ \\
\hline SM $Z W$ & 42 & 0.16 & 0.02 & & 2 & & \\
\hline
\end{tabular}

\begin{tabular}{|c|c|c|c||c|c|c|c|}
\hline $3 \mathrm{TeV}$ & Basic & $M_{\text {eff }}$ & $M_{T}$ & $\mathcal{L}$ & $\#$ Evts & $S / B$ & CL \\
\hline \hline Case (i) & 0.01 & 0.01 & 0.006 & 1000 & 6 & 6 & 0.99 \\
\hline Case (ii) & 0.014 & 0.01 & 0.01 & 1000 & 10 & 10 & $>0.9995$ \\
\hline SM $Z W$ & 42 & 0.05 & 0.001 & & 1 & & \\
\hline
\end{tabular}

$M_{\text {eff }}$ cut: $M_{\text {eff }}>1 \mathrm{TeV}$ (for $M_{W^{\prime}}=2 \mathrm{TeV}$ ) and $M_{\text {eff }}>1.25 \mathrm{TeV}$ (for $M_{W^{\prime}}=3 \mathrm{TeV}$ ).

$M_{T}$ cut: $1.5<M_{T Z W}<2.5 \mathrm{TeV}$ (for $M_{W^{\prime}}=2 \mathrm{TeV}$ ) and $2.4<M_{T Z W}<3.6 \mathrm{TeV}$ (for $\left.M_{W^{\prime}}=3 \mathrm{TeV}\right)$.

In Table 3 we show the cross-sections (in $\mathrm{fb}$ ) for the fully leptonic signal and background for cases (i) and case (ii) with the above cuts applied. Given the small BR into this final state, it is not surprising that we will need a large luminosity to see this signal. The fully leptonic mode is experimentally clean. We find that we need $\mathcal{L}=100 \mathrm{fb}^{-1}\left(\mathcal{L}=1000 \mathrm{fb}^{-1}\right)$ for a $2 \mathrm{TeV}(3 \mathrm{TeV}) W^{\prime}$ to reach a statistically significant signal (in Poisson statistics). We turn next to the semi-leptonic mode which has a larger BR and therefore a larger rate. 
Table 4: The cross-sections (in fb) for the signal process $p p \rightarrow W^{\prime} \rightarrow Z W \rightarrow \ell \ell j j$ for case (i) and case (ii), and SM background, with the cuts applied successively. We show cross-sections for $M_{W^{\prime}}=2$ and $3 \mathrm{TeV}$, and the number of events and significance with the luminosity $\mathcal{L}$ (in $\mathrm{fb}^{-1}$ ) as shown for each case.

\begin{tabular}{|c|c|c|c|c||c|c|c|c|}
\hline $2 \mathrm{TeV}$ & Basic & $M_{\text {eff }}$ & $M_{\text {inv }}$ & $M_{j}$ & $\mathcal{L}$ & \# Evts & $S / B$ & $S / \sqrt{B}$ \\
\hline \hline Case (i) & 0.4 & 0.4 & 0.16 & 0.13 & 1000 & 130 & 0.2 & 5 \\
\hline Case (ii) & 0.5 & 0.48 & 0.38 & 0.3 & 300 & 90 & 0.5 & 6.4 \\
\hline SM $Z W$ & 130 & 0.5 & 0.05 & 0.04 & & 40,12 & & \\
\hline SM $Z+1 \mathrm{j}$ & 3600 & 63 & 2.1 & 0.63 & & 630,190 & & \\
\hline
\end{tabular}

\begin{tabular}{|c|c|c|c|c||c|c|c|c|}
\hline $3 \mathrm{TeV}$ & Basic & $M_{\text {eff }}$ & $M_{\text {inv }}$ & $M_{j}$ & $\mathcal{L}$ & \# Evts & $S / B$ & $S / \sqrt{B}$ \\
\hline \hline Case (i) & 0.03 & 0.03 & 0.01 & - & 1000 & 10 & 0.07 & 0.8 \\
\hline Case (ii) & 0.04 & 0.04 & 0.03 & - & 1000 & 30 & 0.22 & 2.6 \\
\hline SM $Z W$ & 130 & 0.16 & 0.006 & - & & 6 & & \\
\hline SM $Z+1 \mathrm{j}$ & 3600 & 25 & 0.13 & - & & 130 & & \\
\hline
\end{tabular}

\subsubsection{Semi-leptonic channel}

We consider below the two semi-leptonic modes $Z \rightarrow \ell \ell, W \rightarrow j j$ and $Z \rightarrow j j, W \rightarrow \ell \nu$. As explained in detail in Ref. [13, the two jets may merge into one fat jet due to the large boost of the parent gauge boson, picking up a 1-jet background (in addition to the already mentioned SM $Z W$ background). We now consider the signal identification separately.

$Z \rightarrow \ell \ell, W \rightarrow j j$ : Since there is no missing energy in the event we can reconstruct the event fully and form the full invariant mass $\left(M_{i n v}\right.$, not just $\left.M_{T}\right)$. In addition to the SM $Z W$ background, due to jet merging, we have to contend with $Z+1$-jet as a source of background. We apply the following cuts to maximize the signal significance:

$$
\begin{aligned}
& \text { Basic cuts: } p_{T \ell}>250 \mathrm{GeV} ; p_{T j}>500 \mathrm{GeV} ;\left|\eta_{\ell}\right|<2 ;\left|\eta_{j}\right|<2 . \\
& M_{e f f} \text { cut: } M_{e f f}>1 \mathrm{TeV} \text { (for } M_{W^{\prime}}=2 \mathrm{TeV} \text { ) and } M_{e f f}>1.25 \mathrm{TeV} \text { (for } M_{W^{\prime}}=3 \mathrm{TeV} \text { ). } \\
& M_{i n v} \text { cut: } 1.85<M_{Z W}<2.15 \mathrm{TeV} \text { (for } M_{W^{\prime}}=2 \mathrm{TeV} \text { ) and } 2.8<M_{T Z W}<3.2 \mathrm{TeV} \text { (for } \\
& M_{W^{\prime}}=3 \mathrm{TeV} \text { ). }
\end{aligned}
$$$$
\text { Jet-mass cut: } 75<M_{j}<125 \mathrm{GeV} \text {. }
$$

In Table 4 we show the cross-sections as we apply the above cuts successively.

$Z \rightarrow j j, W \rightarrow \ell \nu$ : In addition to the SM $Z W$ background, due to jet merging, we have to contend with $W+1$-jet as a source of background. We apply the following cuts to maximize significance:

Basic cuts: $p_{T \ell}>50 \mathrm{GeV} ; \mathbb{E}_{T}>50 \mathrm{GeV} ;\left|\eta_{\ell}\right|<1 ;\left|\eta_{j}\right|<1$.

$$
M_{\text {eff }} \text { cut: } M_{e f f}>1 \mathrm{TeV} \text { (for } M_{W^{\prime}}=2 \mathrm{TeV} \text { ) and } M_{\text {eff }}>1.25 \mathrm{TeV} \text { (for } M_{W^{\prime}}=3 \mathrm{TeV} \text { ). }
$$


Table 5: The cross-sections (in fb) for the signal process $p p \rightarrow W^{\prime} \rightarrow Z W \rightarrow j j \ell \mathbb{E}_{T}$ for case (i) and case (ii), and SM background, with the cuts applied successively. We show cross-sections for $M_{W^{\prime}}=2$ and $3 \mathrm{TeV}$, and the number of events and significance with the luminosity $\mathcal{L}$ (in $\mathrm{fb}^{-1}$ ) as shown for each case.

\begin{tabular}{|c|c|c|c|c||c|c|c|c|}
\hline $2 \mathrm{TeV}$ & Basic & $M_{\text {eff }}$ & $M_{T}$ & $M_{\text {jet }}$ & $\mathcal{L}$ & $\#$ Evts & $S / B$ & $S / \sqrt{B}$ \\
\hline \hline Case (i) & 1 & 1 & 0.38 & 0.3 & 1000 & 300 & 0.1 & 5.3 \\
\hline Case (ii) & 1.3 & 1.2 & 0.64 & 0.5 & 300 & 150 & 0.16 & 4.9 \\
\hline SM $Z W$ & 320 & 1.2 & 0.04 & 0.03 & & 30,9 & & \\
\hline SM $W+1 \mathrm{j}$ & $3.1 \times 10^{4}$ & 220 & 10.5 & 3.2 & & 3200,950 & & \\
\hline
\end{tabular}

\begin{tabular}{|c|c|c|c|c||c|c|c|c|}
\hline $3 \mathrm{TeV}$ & Basic & $M_{\text {eff }}$ & $M_{T}$ & $M_{\text {jet }}$ & $\mathcal{L}$ & $\#$ Evts & $S / B$ & $S / \sqrt{B}$ \\
\hline \hline Case (i) & 0.08 & 0.08 & 0.016 & - & 1000 & 16 & 0.02 & 0.6 \\
\hline Case (ii) & 0.1 & 0.1 & 0.04 & - & 1000 & 40 & 0.06 & 1.5 \\
\hline SM $Z W$ & 320 & 0.4 & 0.002 & - & & 2 & & \\
\hline SM $W+1 \mathrm{j}$ & $3.1 \times 10^{4}$ & 89 & 0.68 & - & & 680 & & \\
\hline
\end{tabular}

$M_{T}$ cut: $1.8<M_{T Z W}<2.2 \mathrm{TeV}$ (for $M_{W^{\prime}}=2 \mathrm{TeV}$ ) and $2.8<M_{T Z W}<3.2 \mathrm{TeV}$ (for $\left.M_{W^{\prime}}=3 \mathrm{TeV}\right)$.

Jet-mass cut: $75<M_{j}<125 \mathrm{GeV}$.

In Table 5 we show the cross-sections as we apply the above cuts successively.

In the semi-leptonic channels presented above, we find for a $2 \mathrm{TeV} W^{\prime}$ that we need a luminosity of $\mathcal{L}=1000 \mathrm{fb}^{-1}$ and $\mathcal{L}=300 \mathrm{fb}^{-1}$ for cases (i) and (ii) respectively. We thus see that the $t b$ final state discussed earlier which requires about $100 \mathrm{fb}^{-1}$, offers a more promising channel for the $W^{\prime}$ signal observation for case (i) as compared to the semileptonic $\mathrm{W} / \mathrm{Z}$ channels. For a $3 \mathrm{TeV} W^{\prime}$ we find that the QCD background is substantial and the signal-to-background ratio is at a level of a few percent, rendering the signal observation unlikely. Techniques to beat down this reducible QCD background can be beneficial here.

The semi-leptonically decaying neutral electroweak KK gauge boson $\left(Z^{\prime}\right)$ also decays into the $j j \ell \nu$ final state and its detectability has already been discussed in Ref. [13].

\subsection{Wh final state}

Similar to the study in the last section, we again first form the following kinematic variables in order to help separate the signal from background:

$$
\begin{aligned}
M_{e f f_{W h}} & =p_{T W}+p_{T h}, \\
M_{T W h} & =\sqrt{p_{T W}^{2}+M_{W}^{2}}+\sqrt{p_{T h}^{2}+M_{h}^{2}} .
\end{aligned}
$$

In Fig. 8 we show the $M_{e f f_{W h}}$ and $M_{T W h}$ differential distributions for the process $p p \rightarrow W^{+} h$ for the $W^{\prime}$ signal for cases (i) and (ii), and the irreducible SM $W h$ background. We see that the 

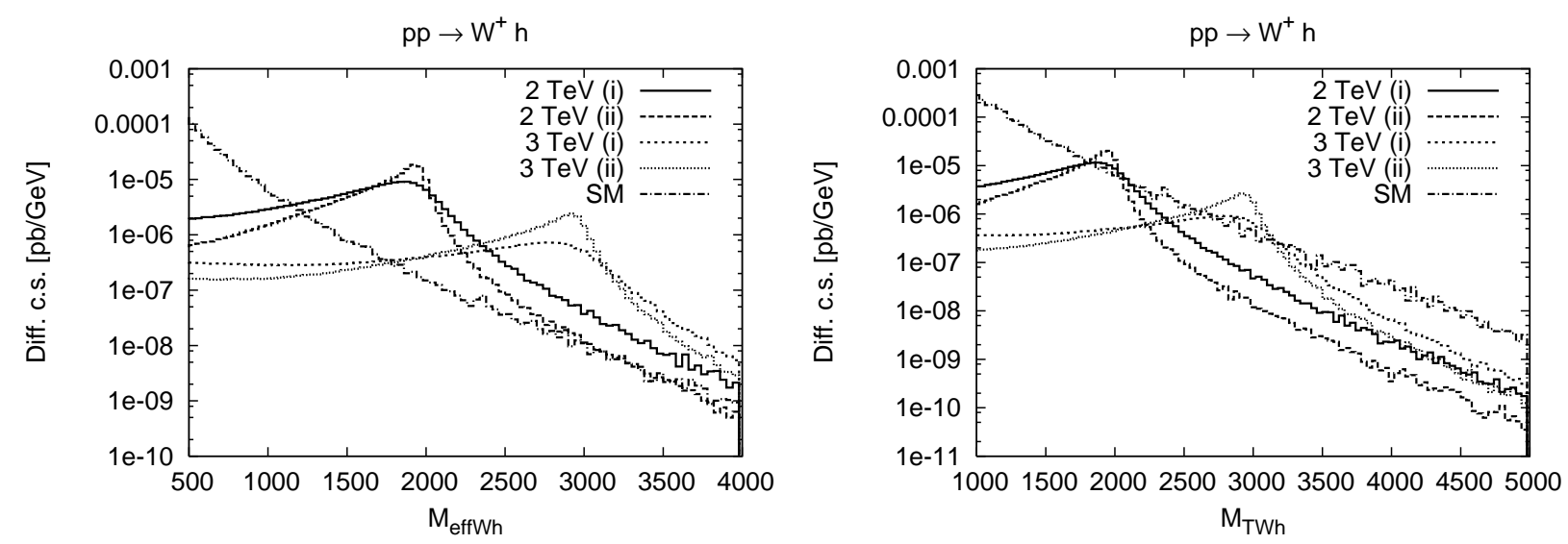

Figure 8: The $M_{\text {eff }}$ (left) and $M_{T W h}$ (right) distributions for signal and SM background for the process $p p \rightarrow W^{+} h$ after the cuts: $p_{T W, h}>100 \mathrm{GeV}$ and $\left|y_{W, h}\right|<3$. Both case (i) and case (ii) are shown.

signal stands comfortably over the background, and with suitably chosen cuts we expect to obtain a good significance. Since $M_{h}$ is unknown, we will take two representative cases: $M_{h}=120 \mathrm{GeV}$ and $150 \mathrm{GeV}$. In the former case the dominant decay mode of the $h$ will be to $b \bar{b}$, while in the latter, to $W^{+} W^{-}$. We will consider each of these cases in turn.

We would like to note that we do not consider the studies here as the Higgs boson discovery channels. Instead, we should consider them as case studies for illustration since we should have had the knowledge about the Higgs properties when our proposed searches are undertaken at the LHC.

\subsection{1 $M_{h}=120 \mathrm{GeV}: h \rightarrow b \bar{b}, W \rightarrow \ell \nu$}

For this case with a relatively low mass, we estimate that $B R(h \rightarrow b \bar{b}) \approx 0.7$. Due to collimation of the decay products of the Higgs, the two b-jets could merge, and we therefore pick-up $W+1$ jet as a source of background. We apply the following cuts to maximize significance:

Basic cuts: $p_{T \ell}>50 \mathrm{GeV} ; \mathbb{E}_{T}>50 \mathrm{GeV} ; p_{T(b b)}>100 \mathrm{GeV} ;\left|\eta_{\ell}\right|<1 ;\left|\eta_{j}\right|<1$.

$M_{\text {eff }}$ cut: $M_{\text {eff }}>1 \mathrm{TeV}$ (for $M_{W^{\prime}}=2 \mathrm{TeV}$ ) and $M_{\text {eff }}>1.25 \mathrm{TeV}$ (for $M_{W^{\prime}}=3 \mathrm{TeV}$ ).

$M_{T}$ cut: $1.8<M_{T W h}<2.2 \mathrm{TeV}$ (for $M_{W^{\prime}}=2 \mathrm{TeV}$ ) and $2.8<M_{T W h}<3.2 \mathrm{TeV}$ (for $\left.M_{W^{\prime}}=3 \mathrm{TeV}\right)$.

b-tag: Due to collimation, we may not be able to resolve the two $b$-jets, and we therefore demand only one b-tag. The efficiency for at least one tagged $b$ is $\epsilon_{b} *\left(2-\epsilon_{b}\right)$. Here, we take the light jet rejection ratio $R_{j}=20$, which, as noted earlier, will likely be improved upon. 
Table 6: The cross-sections (in fb) for the signal process $p p \rightarrow W^{\prime} \rightarrow W h \rightarrow \ell \mathbb{E}_{T} b \bar{b}$ for case (i) and case (ii), and SM background, with the cuts applied successively. We show cross-sections for $M_{W^{\prime}}=2$ and $3 \mathrm{TeV}$, and the number of events and significance with the luminosity $\mathcal{L}$ (in $\mathrm{fb}^{-1}$ ) as shown for each case.

\begin{tabular}{|c|c|c|c|c||c|c|c|c|}
\hline $2 \mathrm{TeV}$ & Basic & $M_{\text {eff }}$ & $M_{T}$ & $\mathrm{~b}$-tag & $\mathcal{L}$ & $\#$ Evts & $S / B$ & $S / \sqrt{B}$ \\
\hline \hline Case (i) & 1.8 & 1.5 & 0.55 & 0.35 & 100 & 35 & 0.65 & 4.8 \\
\hline Case (ii) & 1.6 & 1.5 & 0.8 & 0.5 & 100 & 50 & 1 & 6.4 \\
\hline SM $W h$ & 43 & 0.35 & 0.016 & 0.01 & & 1 & & \\
\hline SM $W+1 \mathrm{j}$ & $3.1 \times 10^{4}$ & 220 & 10.5 & 0.53 & & 53 & & \\
\hline
\end{tabular}

\begin{tabular}{|c|c|c|c|c||c|c|c|c|}
\hline $3 \mathrm{TeV}$ & Basic & $M_{\text {eff }}$ & $M_{T}$ & b-tag & $\mathcal{L}$ & $\#$ Evts & $S / B$ & $C L$ \\
\hline \hline Case (i) & 0.26 & 0.19 & 0.04 & 0.03 & 300 & 9 & 1 & 0.99 \\
\hline Case (ii) & 0.33 & 0.3 & 0.12 & 0.08 & 300 & 24 & 2.4 & $>0.9995$ \\
\hline SM $W h$ & 43 & 0.13 & 0.001 & $6 \times 10^{-4}$ & & 0.2 & & \\
\hline SM $W+1 \mathrm{j}$ & $3.1 \times 10^{4}$ & 89 & 0.68 & 0.03 & & 9 & & \\
\hline
\end{tabular}

In addition to the above cuts, we could apply a jet-mass cut on the collimated $b$-jet system which will peak around $M_{h}$, and can be used to distinguish it from a light-jet. Doing so would improve the significance over that shown here.

In Table 6] we show the cross-sections as we apply the above cuts successively. As expected we find a better significance for case (ii) since the BR is larger. We will need $\mathcal{L}=100 \mathrm{fb}^{-1}$ $\left(\mathcal{L}=300 \mathrm{fb}^{-1}\right)$ for a $2 \mathrm{TeV}(3 \mathrm{TeV}) W^{\prime}$ to reach a good statistical significance. Improving the $b$-tagging performance (by achieving larger $R_{j}$ ) will help reduce the $W+1$ jet background and will better the significance.

\subsection{2 $\quad M_{h}=150 \mathrm{GeV}: h \rightarrow W W \rightarrow \ell \nu j j, W \rightarrow j j$}

For this case with a higher mass, we estimate that $B R(h \rightarrow W W) \approx 0.7$. Due to collimation of the jets from the $W$, we will not demand that separate jets be reconstructed, but rather treat it as a single jet. We will refer to the merged jet closer to the leptonic $W$ as the near-jet $j_{N}$ and the merged jet on the other side as the far-jet $j_{F}$.

We require that there be a jet close to the lepton with $\Delta_{\ell j_{N}}<0.9$, and require $M_{T W j_{N}}$ to be around $M_{h}$, which will reduce the $W+2 j$ background. In addition to the irreducible SM $W h$ background, SM $h+1 j$ also remains as a background, which we will include. In order to differentiate between a light-jet and the $W$-jet we will apply the jet-mass cut as explained in Ref. [13].

We apply the following cuts to maximize significance:

Basic cuts: $p_{T \ell}>25 \mathrm{GeV} ; \mathbb{E}_{T}>25 \mathrm{GeV} ; p_{T j_{N}}>50 \mathrm{GeV} ; p_{T j_{F}}>100 \mathrm{GeV} ;\left|\eta_{\ell}\right|<3 ;$ $\left|\eta_{j_{N}}\right|<3 ;\left|\eta_{j_{F}}\right|<3$.

$M_{\text {eff }}$ cut: $M_{\text {eff }}>1 \mathrm{TeV}$ (for $M_{W^{\prime}}=2 \mathrm{TeV}$ ) and $M_{\text {eff }}>1.25 \mathrm{TeV}$ (for $M_{W^{\prime}}=3 \mathrm{TeV}$ ). 
Table 7: The cross-sections (in fb) for the signal process $p p \rightarrow W^{\prime} \rightarrow W h \rightarrow(j j) W W \rightarrow$ $(j j) \ell \mathbb{E}_{T}(j j)$ for case (i) and case (ii), and SM background, with the cuts applied successively. We show cross-sections for $M_{W^{\prime}}=2$ and $3 \mathrm{TeV}$, and the number of events and significance with the luminosity $\mathcal{L}\left(\right.$ in $\mathrm{fb}^{-1}$ ) as shown for each case.

\begin{tabular}{|c|c|c|c|c||c|c|c|c|}
\hline $2 \mathrm{TeV}$ & Basic & $M_{\text {eff }}$ & $M_{T}$ & $M_{\text {jet }}$ & $\mathcal{L}$ & \# Evts & $S / B$ & CL \\
\hline \hline Case (i) & 1.6 & 1.3 & 0.43 & 0.34 & 100 & 34 & 4 & $\gg 0.9995$ \\
\hline Case (ii) & 2.1 & 1.9 & 0.9 & 0.7 & 100 & 70 & 7 & $\gg 0.9995$ \\
\hline SM $W h$ & 26 & 0.31 & 0.014 & 0.01 & & 1 & & \\
\hline SM $h+1 j$ & 220 & 2 & 0.07 & 0.02 & & 2 & & \\
\hline SM $W+2 j$ & $3 \times 10^{4}$ & 36 & 0.62 & 0.06 & & 6 & & \\
\hline
\end{tabular}

\begin{tabular}{|c|c|c|c|c||c|c|c|c|}
\hline $3 \mathrm{TeV}$ & Basic & $M_{\text {eff }}$ & $M_{T}$ & $M_{\text {jet }}$ & $\mathcal{L}$ & $\#$ Evts & $S / B$ & CL \\
\hline \hline Case (i) & 0.22 & 0.17 & 0.04 & 0.035 & 300 & 11 & 2 & 0.9987 \\
\hline Case (ii) & 0.3 & 0.26 & 0.1 & 0.09 & 300 & 27 & 4 & $\gg 0.9995$ \\
\hline SM $W h$ & 26 & 0.12 & $8 \times 10^{-4}$ & $7 \times 10^{-4}$ & & 0.2 & & \\
\hline SM $h+1 j$ & 220 & 0.72 & $5 \times 10^{-3}$ & $2 \times 10^{-3}$ & & 0.6 & & \\
\hline SM $W+2 j$ & $3 \times 10^{4}$ & 4.1 & 0.05 & 0.015 & & 4.5 & & \\
\hline
\end{tabular}

$M_{T}$ cuts: $100<M_{T W j_{N}}<190 \mathrm{GeV}$ (around $M_{h}$ ); $1.8<M_{T W j_{N} j_{F}}<2.2 \mathrm{TeV}$ (for $M_{W^{\prime}}=$ $2 \mathrm{TeV}$ ) and $2.8<M_{T W j_{N} j_{F}}<3.2 \mathrm{TeV}$ (for $M_{W^{\prime}}=3 \mathrm{TeV}$ ).

Jet-mass cut: $75<M_{j}<125 \mathrm{GeV}$, on both $j_{N}$ and $j_{F}$ for $M_{W^{\prime}}=2 \mathrm{TeV}$, with an acceptance of 0.87 for the $W$-jet and 0.3 for a light-jet [13]. For $M_{W^{\prime}}=3 \mathrm{TeV}$, we apply the jet-mass cut only on $j_{N}$ since its performance in $j_{F}$ might deteriorate owing to increased collimation.

In Table 7 we show the cross-sections as we apply the above cuts successively. Similar to the previous case, we find that we will need $\mathcal{L}=100 \mathrm{fb}^{-1}\left(\mathcal{L}=300 \mathrm{fb}^{-1}\right)$ for a $2 \mathrm{TeV}(3 \mathrm{TeV}) W^{\prime}$ to reach a good statistical significance. The $S / B$ is found to be quite adequate for a signal discovery, and the reach is limited by signal statistics.

\section{4 $W^{\prime} \rightarrow \ell \nu$ final state}

In spite of the unique signal kinematics, we expect the signal event rate to be quite small for this final state given the tiny branching ratio for this mode. Nevertheless, for completeness, we show in Table 8 for $M_{W^{\prime}}=2 \mathrm{TeV}$ the cross-sections for this mode after the following cuts:

Basic cuts: $p_{T \ell}>100 \mathrm{GeV} ; \mathbb{E}_{T}>100 \mathrm{GeV} ;\left|\eta_{\ell}\right|<3$.

$M_{\text {eff }}$ cut: $M_{\text {eff }}>1 \mathrm{TeV}$.

$M_{T}$ cut: $1.5<M_{T \ell \nu}<2.5 \mathrm{TeV}$.

We include the SM $W^{ \pm}$exchange irreducible background. As expected, the signal rate is rather 
Table 8: The cross-sections (in fb) for the signal process $p p \rightarrow W^{\prime} \rightarrow \ell \nu$ for case (i) and case (ii), and SM background, with the cuts applied successively, for $M_{W^{\prime}}=2 \mathrm{TeV}$.

\begin{tabular}{|c|c|c|c|}
\hline $2 \mathrm{TeV}$ & Basic & $M_{\text {eff }}$ & $M_{T}$ \\
\hline \hline Case (i) & 0.04 & 0.024 & 0.012 \\
\hline Case (ii) & 0.05 & 0.04 & 0.02 \\
\hline SM $W$ & $4 \times 10^{3}$ & 6.9 & 0.44 \\
\hline
\end{tabular}

low in comparison with the irreducible SM background. We thus do not expect this mode to be detectable. The modes explored in the previous subsections have much better reach as we have demonstrated.

\section{Comparison to Technicolor Studies}

Based on the AdS/CFT correspondence, the warped extra dimensional model is conjectured to be dual to purely $4 D$ strong dynamics being involved in EWSB, such as technicolor or composite Higgs models. So, we expect similar signals for the two scenarios and therefore it is useful to compare the extensive technicolor studies in the literature (see Ref. [47] for a review) with our current work on signals for electroweak KK gauge bosons in warped extra dimension (including neutral case studied earlier in Ref. [13]).

We begin with the details of this duality which will enable us to compare the signals that we studied to technicolor studies. The $5 D$ model corresponds to a $4 D$ theory with two sector 5 . There is a sector which is strongly coupled, with the strength of the couplings in this sector remaining approximately constant over the Planck-weak hierarchy, i.e., it is a quasi-conformal theory. Conformal invariance is broken at the $\mathrm{TeV}$ scale, resulting in a tower of composite (bound) states starting at $\sim \mathrm{TeV}$ scale. The 2 nd sector consists of particles external to this conformal sector or elementary (as opposed to the composites of the strong sector above). However, these 2 sectors are not isolated, i.e., they do couple to each other. As a result, the elementary particles (external to the CFT sector) mix with the CFT composites and the mass eigenstates (physical states) are admixtures of the two sets of particles. These physical states correspond to the zero and KK modes of the $5 D$ theory.

Furthermore, the location of a mode in the extra dimension is dual to the amount or degree of compositeness (in the sense of the elementary-composite mixture above) of the corresponding state in the $4 D$ theory. Specifically, modes which are localized near the Planck (TeV) brane are interpreted as states which are mostly elementary (composite). Thus, the light SM fermions are mostly elementary, whereas the top quark, Higgs (including unphysical Higgs or longitudinal $W / Z$ )

\footnotetext{
${ }^{5}$ See reference [29] for a two-site description of the $5 D$ model (including the couplings to the heavy new particles) along these lines.
} 
and all KK's are mostly composites (the SM gauge bosons with a flat profile are in-between in terms of compositeness). Roughly speaking, the KK tower of the $5 D$ theory then corresponds to the tower of (massive) composites ("hadrons") in the $4 D$ theory. As discussed earlier, the coupling of a set of modes of the $5 D$ theory is proportional to the overlap of the corresponding profiles in the extra dimension, i.e., it is large if all the modes of this set are localized near the TeV (or Planck) brane and small if some modes are localized near the Planck brane while others are localized near $\mathrm{TeV}$ brane. In the dual $4 D$ theory, the 1st situation correspond to all the particles of the set being mostly composite (or mostly elementary), clearly resulting in a large coupling between these particles, while the 2nd situation involves some particles which are mostly elementary and others which are mostly composites (thus accounting for the small coupling).

We now compare the nature of couplings and hence the decay channels in the warped extra dimensional model that we studied to the case of technicolor theories studied previously. First of all, the decays to physical Higgs bosons $(+W / Z)$ for the electroweak KK's that we studied are new compared to technicolor studies. The reason is that in technicolor theories (at least in the minimal models), there would not be a light Higgs since the idea of technicolor models is that the strong dynamics directly or spontaneously breaks EW symmetry. Equivalently, $W W$ scattering is unitarized by exchange of spin-1 bound states (techni- $\rho$ 's) instead of by a (light) Higgs. On the other hand the warped extra dimensional model that we studied (with a light Higgs in the spectrum) is dual to composite Higgs models in $4 D$, i.e., where strong dynamics does not directly break EW symmetry. Rather, the strong dynamics produces a light composite Higgs which then acquires a vev in the low energy theory to break EW symmetry.

However, the decays of electroweak KK's to $W Z$ or $W W$ and production of KK's in vector boson fusion are (qualitatively) similar to those studied in the technicolor literature for the following reason. Recall that the decays of electroweak KK's to $W Z$ and $W W$ are dominated by longitudinal polarizations of the latter, that too with couplings which are enhanced relative to the SM. Since longitudinal $W / Z$ are equivalent to unphysical Higgs, it is clear (based on the above discussion) that this coupling is dual to a self-coupling of three composites (techni- $\rho$ with composite Goldstones) in the $4 D$ theory and thus is expected to be large. Clearly, such a coupling is a general characteristic of EWSB originating from strong dynamics and is present in all technicolor models studied in the literature. Of course, the details of these couplings at the quantitative level will be different in the $5 D$ model than in the technicolor case (see Ref. [48] for a model-independent parametrization of couplings in composite Higgs models).

For the case of couplings of gauge KK's to fermions, it is convenient to consider two pieces or contributions in the formula for this coupling (see Table 10) as follows. It can be shown that the piece $\propto 1 / \xi$ comes from overlap of profiles near the Planck or UV brane. This part of the coupling is dual to the $\mathrm{SM}$ fermion first coupling to the photon $/ W / Z$ (external to strong dynamics) which 
then mixes with the composite techni- $\rho$ (analog of photon- $\rho$ mixing in QCD). Clearly, this piece of the coupling is present in technicolor models studied in the literature as well and is flavor universal.

The contribution to the coupling of SM fermions to gauge KK's $\propto \xi$ originates from the overlap of profiles near the $\mathrm{TeV}$ or IR brane. In the $4 D$ theory, this part of the coupling corresponds to a direct coupling of SM fermions to the techni- $\rho$, i.e., a coupling involving the composite component of the techni- $\rho$ (as opposed to the coupling via techni- $\rho$ 's mixing with external gauge bosons). Clearly such a contribution arises from (partial) compositeness of the SM fermions themselves and is of similar size to the fermions' coupling to the Higgs (which is another composite). Thus, this piece of the coupling is $\propto 4 D$ or SM Yukawa coupling and is therefore flavor-dependent.

This second contribution to the SM fermion coupling to KK's is absent in "extended technicolor" (ETC), which is the mechanism used in traditional technicolor models to generate fermion masses (instead of partial compositeness of SM fermions as described above). In detail, in ETC, the SM fermion masses originate from the coupling of two SM fermions to (a scalar operator of) strong dynamics such that there is no mixing of external fermions with composite fermions, unlike in the partial compositeness case which involves coupling of a single external fermion to (a fermionic operator of) strong dynamics. In any case, this piece of the coupling is irrelevant for production of gauge KK's via Drell-Yan (DY) process since that involves (dominantly) light fermions, whereas it is relevant for decays of gauge KK's into heavier SM fermions (top/bottom). Therefore, DY production of gauge KK's is (at least qualitatively) similar to that of techni- $\rho$ 's in technicolor, whereas decays to top quarks are different than in the simplest technicolor models with ETC.

In general, using the warped extra dimension framework has the advantage that we have a concrete, weakly coupled model so that we can ensure that we have a consistent set of couplings. In contrast, most technicolor studies simply used a parametrization for the various couplings rather than a well-defined model, although one could conceivably have such a model for these couplings by rescaling QCD data, assuming the strong dynamics is QCD-like.

Other differences between our analyses and earlier studies of technicolor are as follows. Most of the technicolor studies did not go beyond $\sim 2.5 \mathrm{TeV}$ mass for the techni- $\rho$ 's, although the heavier end of the mass range was preferred by constraints from EWPT (specifically the $S$ parameter), while we have considered signals for electroweak KK's up to $3 \mathrm{TeV}$. Finally, the semileptonic decay of the $W W$ or $W Z$ has not been studied in detail in the technicolor context, especially the use of jet mass cut to discriminate a $W / Z$ jet from a $Q C D$ jet.

\section{Discussion and Conclusions}

In the past few years, it has been shown that the framework of a warped extra dimension with SM fields in the bulk can address many of the puzzles of nature. Thus, this framework is a very attractive extension of the SM (perhaps as compelling as SUSY). As the LHC has started, it is 
Table 9: Summary of the best channel for each of the $W^{\prime}$ decay modes, giving the luminosity and significance for the mass shown, in the two $t_{R}$ coupling scenarios of Case (i) and (ii). For the $t b$ channel the numbers without (and with) the reducible $t \bar{t}$ background are shown.

\begin{tabular}{|c|c|c|c|c|}
\hline Case (i): Channel & $M_{W^{\prime}}(\mathrm{TeV})$ & $\mathcal{L}\left(f b^{-1}\right)$ & $S / B$ & $S / \sqrt{B}$ \\
\hline \hline$t b \rightarrow \ell \nu b b$ & 3 & 300 & $5.8(0.9)$ & $0.995(0.95)$ CL \\
\hline$Z W \rightarrow \ell \ell \ell \nu$ & 3 & 1000 & 6 & 0.99 CL \\
\hline$m_{h}=120: W h \rightarrow \ell \nu b b$ & 3 & 300 & 1 & 0.99 CL \\
\hline$m_{h}=150: W h \rightarrow(j j) \ell \nu(j j)$ & 3 & 300 & 2 & 0.9987 CL \\
\hline \hline Case (ii): Channel & $M_{W^{\prime}}(\mathrm{TeV})$ & $\mathcal{L}\left(f b^{-1}\right)$ & $S / B$ & $S / \sqrt{B}$ \\
\hline \hline$t b \rightarrow \ell \nu b b$ & 2 & 1000 & $0.4(0.2)$ & $3.4(2.5) \sigma$ \\
\hline$Z W \rightarrow \ell \ell \ell \nu$ & 3 & 1000 & 10 & $>0.9995 \mathrm{CL}$ \\
\hline$m_{h}=120: W h \rightarrow \ell \nu b b$ & 3 & 300 & 2.4 & $>0.9995 \mathrm{CL}$ \\
\hline$m_{h}=150: W h \rightarrow(j j) \ell \nu(j j)$ & 3 & 300 & 4 & $\gg 0.9995 \mathrm{CL}$ \\
\hline
\end{tabular}

very crucial to study in this framework robust signals from the direct production at the LHC of the new particles, namely the KK excitations of the SM. Over the last year or so, such analyses have been performed for the KK gluon, graviton, $Z$ and some fermions. Here, we continue this program with a study of the charged electroweak KK gauge bosons $\left(W^{\prime}\right)$ thus completing the study of spin-1 states in this framework. We summarize in Table 9 the LHC reach for the two $t_{R}$ cases discussed in Sec. 2.3 with representations shown in Eq. (23), extracting from the detailed analysis we presented in Sec. 4 the best channel for each of the $W^{\prime}$ decay modes. We give the luminosity and resulting significance for the mass shown. We find that we can get a sensitivity of 2 (3) TeV masses with an integrated luminosity of about $100(300) \mathrm{fb}^{-1}$, which although slightly better is comparable to the KK $Z$ reach obtained in Ref. [13].

It is instructive to compare our analysis to the previous ones, starting with various spin- 1 states, in order to illustrate the complementarity of the various studies. The KK gluon has the largest cross-section in this framework, but it decays mostly into $t \bar{t}$ which results in exclusively jetty final states, even if the $W$ from the top decays to leptons (due to the high degree of collimation of decay products of the top quarks). On the other hand, $\mathrm{KK} W$ decays into $W Z$ can result in clean, purely leptonic and fully reconstructible final states, albeit with a small BR which in the end does not result in the reach being larger in this channel 6 In contrast, $\mathrm{KK} Z$ decays into $W W$ can also result in purely leptonic final states, but the invariant mass is not reconstructible in this case. The

\footnotetext{
${ }^{6}$ In more detail, the ability to reconstruct the $W Z$ invariant mass makes the $S / B$ larger, but the effect is diluted a bit when we consider $S / \sqrt{B}$. The effect of a smaller BR of $W Z$ vs. $W W$ to leptons cancels in $S / B$, but still tends to reduce $S / \sqrt{B}$. So, the net result is a significantly larger $S / B$ for the former case, but $S / \sqrt{B}$ is not larger by as much. Another issue is that the KK $W$ can decay into one KK and one zero-mode fermion in the set-ups that we considered (with decays to two light KK fermions being kinematically forbidden in the cases that we study). The presence of this channel dilutes the BR to $W Z$ for the KK $W$. Such a decay channel is suppressed for the KK $Z$ since the light KK fermion comes from a different $5 D$ fermion field than the zero-mode fermion and thus $U(1)$ gauge bosons (including $U(1)$ subgroups of non-abelian gauge multiplets) such as the $Z$ cannot couple these two fermions.
} 
semileptonic analogues of these decays for KK $W / Z$ (i.e., one $W / Z$ decaying leptonically and the other hadronically) are on a similar footing to KK gluon in terms of cleanness since the detection of the highly collimated hadronically decaying $W / Z$ requires discriminating it from the QCD jet background (just like for highly collimated top quarks from decays of the KK gluon): in our analysis a jet mass cut was used for this purpose. Finally, KK $Z$ decays to top pairs are swamped by KK gluon background 7 , but KK $W$ decays to $t \bar{b}$ do not have this problem if the background from KK gluon to $t \bar{t}$ with one highly boosted top faking a bottom can be reduced, for example again by using jet mass as we did here.

We reiterate that further development of techniques for detecting highly boosted $W / Z$ jets and similarly vetoing a highly boosted top faking a bottom can improve the reach for charged (and also neutral) EW states. Another feature we would like to mention is that there are two extreme possibilities for the profiles of top/bottom quarks which are relevant for the KK $W$ search, namely, where the RH or LH top is localized near the so called TeV brane in the extra dimension (while the other chirality has close-to-flat profile) - the point is that either $t_{R}$ or $t_{L}$ must be localized near the $\mathrm{TeV}$ brane in order to obtain the large top mass. The first possibility is favored by flavor precision tests, whereas EW precision tests have a milder preference for the second. Note that the KK modes are also localized near the TeV brane. Hence, the coupling of the KK $W$ (and hence the BR) to LH $t$ (and $\bar{b}$ 焉 is suppressed or enhanced in the two cases and thus vice versa for BR of the other channels with significant couplings to the $\mathrm{KK} W$, i.e., $W Z$ and $W h$, making the two search channels (i.e., $t \bar{b}$ and $W Z / h$ ) complementary in the case of the KK $W$. These two choices for top profiles make less of a difference for the KK $Z$ search since the KK $Z$ always has substantial BR to decay into SM top pairs (of whichever chirality - LH or RH - is localized near the TeV brane).

For a complete perspective of this research program, we now comment on the other spin states. The spin-2 KK graviton is typically heavier than the spin-1 states and thus has an even smaller production cross-section. Its decays to $t \bar{t}$ are not likely to be swamped by those of the KK gluon due to the different mass, but one faces the (even more difficult) challenge of identifying highly boosted top quarks. Decays to $Z Z$ followed by leptons are possibly the cleanest and can moreover be fully reconstructed, but suffer from a very small $\mathrm{BR}$. In contrast, decays to $W W$ cannot be reconstructed in the fully leptonic case (just like in the case of $\mathrm{KK} Z$ ) and challenges for the semileptonic case are similar to $\mathrm{KK} W / Z$ from QCD background.

As far as KK fermions are concerned, the masses of the KK excitations of top/bottom (and their other gauge-group partners) in some models (where the $5 D$ gauge symmetry is extended beyond that in the SM) can be (much) smaller than gauge KK modes, enhancing the prospect for their discovery. In fact, the other heavier (spin-1 or 2) KK modes can decay into these light KK fermions,

\footnotetext{
${ }^{7}$ assuming a small mass splitting between the KK $Z$ and the KK gluon as in the simplest models with no branelocalized kinetic terms for bulk gauge fields

${ }^{8}$ The decays of KK $W$ to $t_{R}$ and $b_{R}$ are usually suppressed since $b_{R}$ is localized near the Planck brane.
} 
resulting in perhaps more distinctive final states for the heavy KK's than the pairs of $W / Z$ or top quarks that have been studied so far - for a recent such study for KK gluon, see Ref. [11. A few studies of signals for the heavier KK fermions and of the radion have also been done. We leave more detailed studies of heavier KK fermions and radion (as well as the rather model-dependent decays of heavier $\mathrm{KK} Z / W /$ graviton into lighter KK's or radion) for future work.

We would like to emphasize that the signals we studied in this paper (and the previous one on neutral gauge bosons) might actually be valid for a wider class of non-supersymmetric models of EWSB. For example, based on AdS/CFT correspondence discussed in Sec. 5, it is clear that any kind of $4 D$ strong dynamics involved in EWSB will (in general) share many of the features of the $5 D$ model. Also, the parameter space of little Higgs models which satisfies EWPT corresponds to the $W^{\prime}, Z^{\prime}$ being weakly coupled to light fermions and strongly coupled to Higgs (including longitudinal $W / Z$ ) [49], just like in the $5 D$ models we studied here. Moreover, some UV completions of little Higgs involve $4 D$ strong dynamics which might have a dual warped extra dimensional description. Thus, little Higgs models and EWSB models with strong dynamics are likely to have LHC signals similar to the ones we have studied. Note however that the flavor structure of the warped extra dimension is different than in traditional technicolor models so that the decays of $\mathrm{KK} W / Z$ to top/bottom are new features. We would also like to point out that the jet mass cut for semileptonic decays of $W Z$ or $W W$ from decay of heavy $W / Z$ has not been studied in detail in these other contexts (technicolor or little Higgs).

In more generality, the point is that there is a class of non-supersymmetric extensions of the SM without a symmetry (analogous to $R$-parity in SUSY) which allow tree-level exchange of new particles to contribute to (purely) SM operators, resulting in strong constraints from precision tests, typically a few $\mathrm{TeV}$ mass for the new particles. Moreover, in many such models, the top/bottom quark and Higgs, including the longitudinal $W / Z$, couple strongly to the new particles since all these particles are closely associated with EWSB. On the other hand, the coupling of the new states to light fermions is typically weak, in part based on considerations of flavor and EW precision tests. Thus, a large class of non-supersymmetric models faces challenges similar to the warped extra dimension framework that we studied here, namely, production of the new states tends to be suppressed and decays are mostly to top quarks $/ W / Z$, that too highly boosted. In summary, the techniques we developed in this paper might be useful for obtaining signals for a wider class of models, beyond warped extra dimensions.

\section{Acknowledgments}

We would like to thank H. Davoudiasl, D. E. Kaplan, W. Kilgore, F. Paige, G. Perez, Z. Si, C. Sturm, M. Strassler and R. Sundrum for discussions, and, A. Belyaev for help with CalcHEP, and S. Mrenna and P. Skands for help with Pythia. KA is supported in part by NSF grant No. 
PHY-0652363. SG and AS are supported in part by the DOE grant DE-AC02-98CH10886 (BNL). TH and G.-Y.H are supported in part by a DOE grant DE-FG02-95ER40896 and in part by the Wisconsin Alumni Research Foundation, and G.-Y.H is also supported by DOE grant DE-FG0291ER40674 and by the U.C. Davis HEFTI program.

\section{A Couplings and Mixing Angles}

Here we collect from Ref. [13, expressions for couplings and mixing angles. We focus mainly on the fermion representation with the custodial symmetry protecting $Z b \bar{b}$. For our numerical study, we assume $g_{L}=g_{R}$ throughout. The mixing angles and couplings are related through (with $s \equiv \sin ()$ and $c \equiv \cos ())$

$$
\begin{aligned}
g^{\prime} & =\frac{g_{X} g_{R}}{\sqrt{g_{R}^{2}+g_{X}^{2}}}, \quad s^{\prime}=\frac{g_{X}}{\sqrt{g_{R}^{2}+g_{X}^{2}}}, \quad c^{\prime}=\sqrt{1-s^{\prime 2}}, \\
e & =\frac{g_{L} g^{\prime}}{\sqrt{g^{\prime 2}+g_{L}^{2}}}, \quad s_{W}=\frac{g^{\prime}}{\sqrt{g^{\prime 2}+g_{L}^{2}}}, \quad c_{W}=\sqrt{1-s_{W}^{2}}, \\
g_{Z} & =g_{L} / c_{W}, \quad g_{Z^{\prime}}=g_{R} / c^{\prime} .
\end{aligned}
$$

For the case $g_{R}=g_{L}$, we have $s^{\prime}=0.55, c^{\prime}=0.84$.

The various mixing angles are as explained in Ref. [13] and we repeat below a few relevant ones. As explained in Apps. A and B of Ref. [13, EWSB induces a mixing between $Z^{(0)} \leftrightarrow Z_{1}$ (with mixing angle $\theta_{01}$ ) and $Z^{(0)} \leftrightarrow Z_{X_{1}}$ (with mixing angle $\theta_{01 X}$ ). To leading order in $M_{Z} / M_{Z^{\prime}}$ these mixing angles are given by

$$
\begin{array}{r}
\sin \theta_{01} \approx\left(\frac{M_{Z}}{M_{Z_{1}}}\right)^{2} \sqrt{k \pi r_{c}}, \\
\sin \theta_{01 X} \approx-\left(\frac{M_{Z}}{M_{Z_{X 1}}}\right)^{2}\left(\frac{g_{Z^{\prime}}}{g_{Z}}\right) c^{\prime 2} \sqrt{k \pi r_{c}} .
\end{array}
$$

For example, for $M_{Z^{\prime}}=2 \mathrm{TeV}, s_{01}=0.013$ and $s_{01 X}=-0.01$.

EWSB similarly induces mixing in the charged $W^{ \pm}$sector i.e. mixing between $W \leftrightarrow W^{\prime}$, with mixing angle given by

$$
\begin{array}{r}
\sin \theta_{0 L} \approx\left(\frac{M_{W}}{M_{W_{L_{1}}}}\right)^{2} \sqrt{k \pi r_{c}}, \\
\sin \theta_{0 R} \approx-\left(\frac{M_{W}}{M_{W_{R_{1}}}}\right)^{2}\left(\frac{g_{R}}{g_{L}}\right) \sqrt{k \pi r_{c}} .
\end{array}
$$

For example, for $M_{Z^{\prime}}=2 \mathrm{TeV}, s_{0 L} \approx 0.01$ and $s_{0 R} \approx-0.01$.

EWSB also induces $Z_{1} \leftrightarrow Z_{X 1}$ mixing, with mixing angle given by

$$
\tan 2 \theta_{1}=\frac{-2 M_{Z}^{2}\left(g_{Z^{\prime}} / g_{Z}\right) c^{\prime 2} k \pi r_{c}}{\left(M_{Z_{X_{1}}}^{2}-M_{Z_{1}}^{2}\right)+M_{Z}^{2}\left(\left(g_{Z^{\prime}} / g_{Z}\right)^{2} c^{4}-1\right) k \pi r_{c}}
$$


For example, for $M_{Z_{1}}=2000 ; M_{Z_{X 1}}=1962 \mathrm{GeV}$, this implies that $s_{1}=0.48, c_{1}=0.88$. After this mixing, we will refer to the mass eigenstates as $\tilde{Z}_{1}$ and $\tilde{Z}_{X 1}$.

EWSB similarly induces $W_{L_{1}} \leftrightarrow W_{R_{1}}$ with mixing angle given by

$$
\tan 2 \theta_{1}^{c}=\frac{-2 M_{W}^{2}\left(g_{R} / g_{L}\right) k \pi r_{c}}{\left(M_{W_{R_{1}}}^{2}-M_{W_{L_{1}}}^{2}\right)+M_{W}^{2}\left(\left(g_{R} / g_{L}\right)^{2}-1\right) k \pi r_{c}} .
$$

For example, for $M_{W_{L_{1}}}=2000 ; M_{W_{R_{1}}}=1962 \mathrm{GeV}$, this implies that $s_{1}^{c}=0.6, c_{1}^{c}=0.8$. After this mixing, we will refer to the mass eigenstates as $\tilde{W}_{L_{1}}$ and $\tilde{W}_{R_{1}}$, and for notational easy we will just denote them as $W_{L}^{\prime}$ and $W_{R}^{\prime}$ respectively.

\section{B Couplings of $W^{\prime}$}

The electroweak gauge group in the bulk is $S U(2)_{L} \times S U(2)_{R} \times U(1)_{X}$, with hypercharge being a linear combination of $U(1)_{R}$ and $U(1)_{X}$. The extra $S U(2)_{R}$ (relative to the SM) ensures suppression of contribution to the EWPT (specifically an observable called the $T$ parameter). Hence, we obtain 2 charged KK towers (before EWSB) - one from each $S U(2)$ group in the bulk. We will restrict to the 1 st KK modes only in each tower and denote them by $W_{L 1}$ and $W_{R 1}$, respectively. EWSB mixes the 2 and the resulting mass eigenstates are denoted by $\tilde{W}_{R 1}$ and $\tilde{W}_{L 1}$.

We work in the approximation $\left(k \pi r_{c}\right) m_{W}^{2} / M_{K K}^{2} \ll 1$.

\section{B.1 $W^{\prime}$ coupling to fermions}

We show below the fermion representations under $S U(2)_{L} \otimes S U(2)_{R} \otimes U(1)_{X}$, denoted as $(L, R)_{X}$. We take the left handed quarks of the first and second generation, and the left-handed leptons to be doublets under $S U(2)_{L}$. This specifies the interaction of these fields with $W_{L_{1}}$. The $W_{R_{1}}$ couplings to first and second generation quarks, right-handed bottom quark and leptons are negligibly small since the $W_{R_{1}}$ profile is suppressed near the Planck brane where these fermion fields are peaked. To have the custodial symmetry protection of the $Z b \bar{b}$ coupling [37], we take the third generation left-handed quarks to be in the representation

$$
Q_{L}^{3}=\left(\begin{array}{ll}
q_{L}^{3} & q_{L}^{\prime}
\end{array}\right)=\left(\begin{array}{cc}
t_{L} & \chi_{L} \\
b_{L} & T_{L}
\end{array}\right) \rightarrow(2,2)_{2 / 3}
$$

where $\chi_{L}, T_{L}$ are taken to have $(-+)$ boundary conditions $(\mathrm{BC})$ with no zero-modes 9 . We have $Q\left(\chi_{L}\right)=5 / 3$ and $Q\left(T_{L}\right)=2 / 3$. To accommodate the large top and bottom mass difference we take it that $t_{R}$ and $b_{R}$ do not belong to the same $S U(2)_{R}$ multiplet. We consider two cases for the

\footnotetext{
${ }^{9}$ All SM fermions have $(++) \mathrm{BC}$ since they are zero modes.
} 
$t_{R}$ representations

$$
\begin{array}{ll}
\text { Case(i) } \quad: \quad t_{R} \rightarrow(1,1)_{2 / 3}, \\
\text { Case(ii) } \quad: \quad t_{R} \rightarrow(1,3)_{2 / 3} \oplus(3,1)_{2 / 3}=\left(\begin{array}{c}
\chi_{R}^{\prime \prime} \\
t_{R} \\
B_{R}^{\prime \prime}
\end{array}\right) \oplus\left(\begin{array}{c}
\chi_{R}^{\prime \prime \prime} \\
T_{R}^{\prime \prime \prime} \\
B_{R}^{\prime \prime \prime}
\end{array}\right),
\end{array}
$$

where the exotic fermions have $(-+) \mathrm{BC}$ with no zero-modes, and the fermions in the $(3,1)$ representation are not discussed further in our work here since the $W^{\prime}$ decay to a pair of them is kinematically forbidden. For Case $(\mathrm{i}), t_{R} \rightarrow(1,1)$, the electroweak precision tests (EWPT) are better satisfied [38] for $c_{Q_{L}^{3}}=0$ and $c_{t_{R}}=0.4$, i.e., $Q_{L}^{3}$ peaked closer to the $\mathrm{TeV}$ brane, while for Case (ii), $t_{R} \rightarrow(1,3)$, for $c_{Q_{L}^{3}}=0.4$ and $c_{t_{R}}=0$, i.e., $c_{t_{R}}$ peaked closer to the TeV brane. After including the charges and the overlap integrals, the largest effective coupling of third generation fermions to gauge KK modes in Case (i) would be to $Q_{L}^{3}$, being larger than that in Case (ii), which would be to $t_{R}$. Consequently, while on the one hand new gauge KK induced FCNC contributions would be larger in Case (i) and hence more problematic for the simplest constructions, on the other hand collider signals would be larger compared to Case (ii).

The fermion couplings to $W^{\prime}$ depend on various mixing angles summarized in App. A. The couplings also depend on the overlap integrals which we give next. We note that $W_{L_{1}}$ has $(++)$ while $W_{R_{1}}$ has $(-+)$ BC. The overlap integrals of a $W^{\prime}$ with two fermions are given in Table 10, We represent by $\mathcal{I}_{y y, z z}^{x x}$ the overlap integral of the $W^{\prime}$ having $x x \mathrm{BC}$ with two fermion fields one with $y y$ and the other with $z z$ BC's. For instance, $\mathcal{I}_{++,++}^{++}$is the overlap integral of the $W_{L_{1}}$ with two fermions both with $(++) \mathrm{BC}$, and, $\mathcal{I}_{++,-+}^{++}$the overlap integral of the $W_{L_{1}}$ with one fermions with $(++)$ BC and the other with $(-+)$ BC. Similarly, $\mathcal{I}_{y y, z z}^{-+}$represents the overlap integral of the $W_{R_{1}}$ with two fermions. Due to the orbifold $Z_{2}$ symmetry, we have $I_{++,-+}^{++}=0$ etc., and we show only the nonzero ones in the table.

We note that the mass of the $(-+)$ fermion is lighter than $M_{\tilde{W}_{L_{1}}}$ for $c<1 / 2$. In particular for $c=0.4$ it is about $0.9 M_{\tilde{W}_{L_{1}}}$, and for $c=0$ it is about $0.6 M_{\tilde{W}_{L_{1}}}$. The first KK excitation of the $(++)$ fermions are typically heavier than $M_{\tilde{W}_{L_{1}}}$ (being equal at $c=1 / 2$ ).

The $W^{\prime}$ coupling (Feynman rule) to fermions is given by

$$
\overline{u_{L}} d_{L}\left\{\tilde{W}_{L_{1}}^{+}, \tilde{W}_{R_{1}}^{+}\right\}: i \frac{g_{L}}{\sqrt{2}}\left\{c_{1}^{c}, s_{1}^{c}\right\} \mathcal{I}_{u_{L} d_{L}}^{++}
$$

where $u_{L}\left(d_{L}\right)$ denotes first and second generation up- (down-) type fermions. The third generation 
Table 10: Values of $\psi \psi W^{\prime}$ overlap integrals for: Case (i), $t_{R} \rightarrow(1,1), c_{Q_{L}^{3}}=0$ and $c_{t_{R}}=0.4$ (upper table), and, Case (ii), $t_{R} \rightarrow(1,3), c_{Q_{L}^{3}}=0.4$ and $c_{t_{R}}=0$ (lower table). All the other $c$ 's $>0.5$. We take $\xi=\sqrt{k \pi r_{c}}=5$.83. All SM fermions have $(++)$ BC, "exotic" BSM fermions have $(-+), W_{L_{1}}$ has $(++)$, and, $W_{R_{1}}$ has $(-+)$ BC.

\begin{tabular}{|c||c|c|c|}
\hline$c_{Q_{L}^{3}}=0, c_{t_{R}}=0.4$ & $Q_{L}^{3}$ & $t_{R}$ & other fermions \\
\hline \hline $\mathcal{I}_{++,++}^{++}$ & $-\frac{1.13}{\xi}+0.7 \xi \approx 3.9$ & $-\frac{1.13}{\xi}+0.2 \xi \approx 1$ & $-\frac{1.13}{\xi} \approx-0.2$ \\
\hline $\mathcal{I}_{-+,-+}^{++}$ & $\xi$ & $\xi$ & - \\
\hline $\mathcal{I}_{++,-+}^{-+}$ & $0.8 \xi \approx 4.6$ & $0.4 \xi \approx 2.3$ & $\approx 0$ \\
\hline \hline$c_{Q_{L}^{3}}=0.4, c_{t_{R}}=0$ & $Q_{L}^{3}$ & $t_{R}$ & other fermions \\
\hline \hline $\mathcal{I}_{++,++}^{++}$ & $-\frac{1.13}{\xi}+0.2 \xi \approx 1$ & $-\frac{1.13}{\xi}+0.7 \xi \approx 3.9$ & $-\frac{1.13}{\xi} \approx-0.2$ \\
\hline $\mathcal{I}_{-+,-+}^{++}$ & $\xi$ & $\xi$ & - \\
\hline $\mathcal{I}_{++,-+}^{-+}$ & $0.4 \xi \approx 2.3$ & $0.8 \xi \approx 4.6$ & $\approx 0$ \\
\hline
\end{tabular}

left-handed fermion couplings are give by

$$
\begin{array}{ccc}
\overline{t_{L}} b_{L}\left\{\tilde{W}_{L_{1}}^{+}, \tilde{W}_{R_{1}}^{+}\right\} & : & i \frac{g_{L}}{\sqrt{2}}\left\{c_{1}^{c}, s_{1}^{c}\right\} \mathcal{I}_{t_{L} b_{L}}^{++}, \\
\overline{\chi_{L}} T_{L}\left\{\tilde{W}_{L_{1}}^{+}, \tilde{W}_{R_{1}}^{+}\right\} & : & i \frac{g_{L}}{\sqrt{2}}\left\{c_{1}^{c}, s_{1}^{c}\right\} \mathcal{I}_{\chi_{L} T_{L}}^{++}, \\
\overline{\chi_{L}} t_{L}\left\{\tilde{W}_{L_{1}}^{+}, \tilde{W}_{R_{1}}^{+}\right\} & : & i \frac{g_{R}}{\sqrt{2}}\left\{s_{1}^{c},-c_{1}^{c}\right\} \mathcal{I}_{\chi_{L} t_{L}}^{-+} \\
\overline{T_{L}} b_{L}\left\{\tilde{W}_{L_{1}}^{+}, \tilde{W}_{R_{1}}^{+}\right\} & : & i \frac{g_{R}}{\sqrt{2}}\left\{s_{1}^{c},-c_{1}^{c}\right\} \mathcal{I}_{T_{L} b_{L}}^{-+}
\end{array}
$$

For Case (i), $t_{R} \rightarrow(1,1)$, it does not interact with the $W^{\prime}$ as already mentioned. For Case (ii), $t_{R} \rightarrow(1,3)$ its interaction with the $W^{\prime}$ is given as

$$
\begin{array}{ccc}
\overline{\chi_{R}^{\prime \prime}} t_{R}\left\{\tilde{W}_{L_{1}}^{+}, \tilde{W}_{R_{1}}^{+}\right\} & : i \frac{g_{R}}{2}\left\{-s_{1}^{c}, c_{1}^{c}\right\} \mathcal{I}_{\chi_{R}^{\prime \prime} t_{R}}^{-+}, \\
\overline{t_{R}} B_{R}^{\prime \prime}\left\{\tilde{W}_{L_{1}}^{+}, \tilde{W}_{R_{1}}^{+}\right\} & : & i \frac{g_{R}}{2}\left\{-s_{1}^{c}, c_{1}^{c}\right\} \mathcal{I}_{t_{R} B_{R}^{\prime \prime}}^{-+} .
\end{array}
$$

\section{B.2 $W^{\prime}$ coupling to two SM gauge bosons}

In order to derive the triple gauge boson coupling we start with the KK basis Lagrangian terms (keeping in mind $W_{R}^{ \pm(0)} \equiv 0$ )

$\mathcal{L} \supset-g_{L} W_{L}^{3(0)} W_{L}^{+(0)} W_{L}^{-(0)}-g_{L} W_{L}^{3(1)} W_{L}^{+(1)} W_{L}^{-(0)}-g_{L} W_{L}^{3^{(0)}} W_{L}^{+(1)} W_{L}^{-(1)}-g_{R} W_{R}^{3(0)} W_{R}^{+(1)} W_{R}^{-(1)}$.

Writing this in the mass eigenbasis results in the triple gauge boson couplings (Feynman rules).

The $A W^{\prime \pm} W^{\mp}$ coupling is zero. The $Z$ couplings (Feynman rules) are given by

$$
\begin{array}{lll}
Z \tilde{W}_{L_{1}}^{+} W^{-} & : & -i g_{L} c_{W}\left[-s_{0 R} s_{1}^{c}\left(\frac{g_{R}}{g_{L}} \frac{s_{W}}{c_{W}} s^{\prime}+1\right)-s_{01} c_{1}^{c}\right], \\
Z \tilde{W}_{R_{1}}^{+} W^{-} & : & -i g_{L} c_{W}\left[s_{0 R} c_{1}^{c}\left(\frac{g_{R}}{g_{L}} \frac{s_{W}}{c_{W}} s^{\prime}+1\right)-s_{01} s_{1}^{c}\right],
\end{array}
$$


and for comparison we note that the SM triple gauge boson coupling is given as

$$
\{A, Z\} W^{+} W^{-}:-i g_{L}\left\{s_{W}, c_{W}\right\} \text {. }
$$

\section{B.3 $W^{\prime}$ coupling to $W$ and Higgs}

Starting from Eq. (44) of Ref. [13] we obtain the couplings to the Higgs by making the substitution $v \rightarrow(v+h)$ which results in the couplings (Feynman rules)

$$
\begin{aligned}
\left\{\tilde{W}_{L_{1}}^{+}, \tilde{W}_{R_{1}}^{+}\right\} W^{-} h & : i \frac{2 m_{W}^{2}}{v} \sqrt{k \pi r_{c}}\left\{\left(c_{1}^{c}+\frac{g_{R}}{g_{L}} s_{1}^{c}\right),\left(s_{1}^{c}-\frac{g_{R}}{g_{L}} c_{1}^{c}\right)\right\} \\
\left\{\tilde{W}_{L_{1}}^{+}, \tilde{W}_{R_{1}}^{+}\right\} W^{-} h h & : i \frac{2 m_{W}^{2}}{v^{2}} \sqrt{k \pi r_{c}}\left\{\left(c_{1}^{c}+\frac{g_{R}}{g_{L}} s_{1}^{c}\right),\left(s_{1}^{c}-\frac{g_{R}}{g_{L}} c_{1}^{c}\right)\right\}
\end{aligned}
$$

where the $h h$ couplings include a symmetry factor of 2 .

\section{References}

[1] L. Randall and R. Sundrum, Phys. Rev. Lett. 83, 3370 (1999) arXiv:hep-ph/9905221.

[2] H. Davoudiasl, J. L. Hewett and T. G. Rizzo, Phys. Lett. B 473, 43 (2000) arXiv:hep-ph/9911262; A. Pomarol, Phys. Lett. B 486, 153 (2000) arXiv:hep-ph/9911294; S. Chang, J. Hisano, H. Nakano, N. Okada and M. Yamaguchi, Phys. Rev. D 62, 084025 (2000) arXiv:hep-ph/9912498.

[3] Y. Grossman and M. Neubert, Phys. Lett. B 474, 361 (2000) arXiv:hep-ph/9912408.

[4] T. Gherghetta and A. Pomarol, Nucl. Phys. B 586, 141 (2000) arXiv:hep-ph/0003129.

[5] K. Agashe, R. Contino and R. Sundrum, Phys. Rev. Lett. 95, 171804 (2005) arXiv:hep-ph/0502222.

[6] K. Agashe and G. Servant, Phys. Rev. Lett. 93, 231805 (2004) arXiv:hep-ph/0403143 and JCAP 0502, 002 (2005) arXiv:hep-ph/0411254.

[7] T. G. Rizzo, JHEP 0206, 056 (2002) arXiv:hep-ph/0205242; C. Csaki, J. Hubisz and S. J. Lee, Phys. Rev. D 76, 125015 (2007) arXiv:0705.3844 [hep-ph]]; M. Toharia, arXiv:0809.5245 [hepph].

[8] K. Agashe, A. Belyaev, T. Krupovnickas, G. Perez and J. Virzi, Phys. Rev. D 77, 015003 (2008) arXiv:hep-ph/0612015.

[9] B. Lillie, L. Randall and L. T. Wang, JHEP 0709, 074 (2007) arXiv:hep-ph/0701166. 
[10] M. Guchait, F. Mahmoudi and K. Sridhar, JHEP 0705, 103 (2007) arXiv:hep-ph/0703060; B. Lillie, J. Shu and T. M. P. Tait, Phys. Rev. D 76, 115016 (2007) [arXiv:0706.3960 [hep-ph]]; A. Djouadi, G. Moreau and R. K. Singh, Nucl. Phys. B 797, 1 (2008) arXiv:0706.4191 [hepph]]; M. Guchait, F. Mahmoudi and K. Sridhar, Phys. Lett. B 666, 347 (2008) arXiv:0710.2234 [hep-ph]].

[11] M. Carena, A. D. Medina, B. Panes, N. R. Shah and C. E. M. Wagner, Phys. Rev. D 77, 076003 (2008) arXiv:0712.0095 [hep-ph]].

[12] A. L. Fitzpatrick, J. Kaplan, L. Randall and L. T. Wang, JHEP 0709, 013 (2007) arXiv:hep-ph/0701150]; K. Agashe, H. Davoudiasl, G. Perez and A. Soni, Phys. Rev. D 76, 036006 (2007) arXiv:hep-ph/0701186; O. Antipin, D. Atwood and A. Soni, Phys. Lett. B 666, 155 (2008) arXiv:0711.3175 [hep-ph]]; O. Antipin and A. Soni, arXiv:0806.3427 [hep-ph].

[13] K. Agashe et al., Phys. Rev. D 76, 115015 (2007) [arXiv:0709.0007 [hep-ph]].

[14] H. Davoudiasl, T. G. Rizzo and A. Soni, Phys. Rev. D 77, 036001 (2008) arXiv:0710.2078 [hep-ph]].

[15] C. Dennis, M. Karagoz Unel, G. Servant and J. Tseng, arXiv:hep-ph/0701158; R. Contino and G. Servant, JHEP 0806, 026 (2008) [arXiv:0801.1679 [hep-ph]].

[16] H. Davoudiasl, J. L. Hewett and T. G. Rizzo, Phys. Rev. Lett. 84, 2080 (2000) arXiv:hep-ph/9909255]; H. Davoudiasl, J. L. Hewett and T. G. Rizzo, Phys. Rev. D 63, 075004 (2001) arXiv:hep-ph/0006041; S. C. Park, H. S. Song and J. H. Song, Phys. Rev. D 65, 075008 (2002) arXiv:hep-ph/0103308; H. Davoudiasl and T. G. Rizzo, Phys. Lett. B 512, 100 (2001) arXiv:hep-ph/0104199; H. Davoudiasl, J. L. Hewett, B. Lillie and T. G. Rizzo, Phys. Rev. D 70, 015006 (2004) arXiv:hep-ph/0312193; A. Birkedal, K. Matchev and M. Perelstein, Phys. Rev. Lett. 94 (2005) 191803 arXiv:hep-ph/0412278; F. Ledroit, G. Moreau and J. Morel, arXiv:hep-ph/0703262; A. Djouadi and G. Moreau, arXiv:0707.3800 [hep-ph]; M. Piai, arXiv:0704.2205 [hep-ph].

[17] R. Contino, Y. Nomura and A. Pomarol, Nucl. Phys. B 671, 148 (2003) arXiv:hep-ph/0306259.

[18] W. D. Goldberger and M. B. Wise, Phys. Rev. Lett. 83, 4922 (1999) arXiv:hep-ph/9907447; J. Garriga and A. Pomarol, Phys. Lett. B 560, 91 (2003) arXiv:hep-th/0212227.

[19] J. M. Maldacena, Adv. Theor. Math. Phys. 2, 231 (1998) [Int. J. Theor. Phys. 38, 1113 (1999)] arXiv:hep-th/9711200]; S. S. Gubser, I. R. Klebanov and A. M. Polyakov, Phys. Lett. 
B 428, 105 (1998) arXiv:hep-th/9802109]; E. Witten, Adv. Theor. Math. Phys. 2, 253 (1998) arXiv:hep-th/9802150.

[20] N. Arkani-Hamed, M. Porrati and L. Randall, JHEP 0108, 017 (2001) arXiv:hep-th/0012148;

R. Rattazzi and A. Zaffaroni, JHEP 0104, 021 (2001) arXiv:hep-th/0012248.

[21] S. J. Huber and Q. Shafi, Phys. Lett. B 498, 256 (2001) arXiv:hep-ph/0010195.

[22] K. Agashe, G. Perez and A. Soni, Phys. Rev. D 71, 016002 (2005) arXiv:hep-ph/0408134.

[23] C. Csaki, A. Falkowski and A. Weiler, JHEP 0809, 008 (2008) arXiv:0804.1954 [hep-ph]]; M. Blanke, A. J. Buras, B. Duling, S. Gori and A. Weiler, arXiv:0809.1073 [hep-ph].

[24] A. L. Fitzpatrick, G. Perez and L. Randall, arXiv:0710.1869 [hep-ph].

[25] S. Davidson, G. Isidori and S. Uhlig, Phys. Lett. B 663, 73 (2008) arXiv:0711.3376 [hep-ph]].

[26] H. Davoudiasl, B. Lillie and T. G. Rizzo, JHEP 0608, 042 (2006) arXiv:hep-ph/0508279. M. Piai, arXiv:hep-ph/0608241; G. Cacciapaglia, C. Csaki, G. Marandella and J. Terning, JHEP 0702, 036 (2007) arXiv:hep-ph/0611358; J. Hirn and V. Sanz, JHEP 0703, 100 (2007) arXiv:hep-ph/0612239; C. D. Carone, J. Erlich and J. A. Tan, Phys. Rev. D 75, 075005 (2007) arXiv:hep-ph/0612242.

[27] P. McGuirk, G. Shiu and K. M. Zurek, JHEP 0803, 012 (2008) arXiv:0712.2264 [hep-ph]]; G. Shiu, B. Underwood, K. M. Zurek and D. G. E. Walker, Phys. Rev. Lett. 100, 031601 (2008) [arXiv:0705.4097 [hep-ph]]; A. Falkowski and M. Perez-Victoria, arXiv:0806.1737 [hepph]; B. Batell, T. Gherghetta and D. Sword, arXiv:0808.3977 [hep-ph].

[28] M. Carena, E. Ponton, T. M. P. Tait and C. E. M. Wagner, Phys. Rev. D 67, 096006 (2003) arXiv:hep-ph/0212307]; H. Davoudiasl, J. L. Hewett and T. G. Rizzo, Phys. Rev. D 68, 045002 (2003) arXiv:hep-ph/0212279; M. S. Carena, A. Delgado, E. Ponton, T. M. P. Tait and C. E. M. Wagner, Phys. Rev. D 68, 035010 (2003) arXiv:hep-ph/0305188]; M. S. Carena, A. Delgado, E. Ponton, T. M. P. Tait and C. E. M. Wagner, Phys. Rev. D 71, 015010 (2005) arXiv:hep-ph/0410344.

[29] R. Contino, T. Kramer, M. Son and R. Sundrum, JHEP 0705, 074 (2007) arXiv:hep-ph/0612180.

[30] K. Agashe, A. Azatov and L. Zhu, arXiv:0810.1016 [hep-ph].

[31] G. Cacciapaglia, C. Csaki, J. Galloway, G. Marandella, J. Terning and A. Weiler, JHEP 0804, 006 (2008) arXiv:0709.1714 [hep-ph]]; M. C. Chen and H. B. Yu, arXiv:0804.2503 [hepph]; G. Perez and L. Randall, arXiv:0805.4652 [hep-ph]; C. Csaki, C. Delaunay, C. Grojean 
and Y. Grossman, arXiv:0806.0356 [hep-ph]; J. Santiago, arXiv:0806.1230 [hep-ph]; C. Csaki, A. Falkowski and A. Weiler, arXiv:0806.3757 [hep-ph]; C. Csaki, Y. Grossman, G. Perez, Z. Surujon and A. Weiler, to appear.

[32] H. Davoudiasl, G. Perez and A. Soni, Phys. Lett. B 665, 67 (2008) [arXiv:0802.0203 [hep-ph]].

[33] P. M. Aquino, G. Burdman and O. J. P. Eboli, Phys. Rev. Lett. 98, 131601 (2007) arXiv:hep-ph/0612055.

[34] For studies with $10 \mathrm{TeV}$ KK masses, see S. J. Huber, Nucl. Phys. B 666, 269 (2003) arXiv:hep-ph/0303183; S. Khalil and R. Mohapatra, Nucl. Phys. B 695, 313 (2004) arXiv:hep-ph/0402225.

[35] G. Burdman, Phys. Lett. B 590, 86 (2004) arXiv:hep-ph/0310144; K. Agashe, G. Perez and A. Soni, Phys. Rev. Lett. 93, 201804 (2004) arXiv:hep-ph/0406101]; G. Moreau and J. I. SilvaMarcos, JHEP 0603, 090 (2006) arXiv:hep-ph/0602155; K. Agashe, A. E. Blechman and F. Petriello, Phys. Rev. D 74, 053011 (2006) arXiv:hep-ph/0606021]; K. Agashe, G. Perez and A. Soni, Phys. Rev. D 75, 015002 (2007) arXiv:hep-ph/0606293]; S. Chang, C. S. Kim and J. Song, JHEP 0702, 087 (2007) arXiv:hep-ph/0607313 and Phys. Rev. D 77, 075001 (2008) arXiv:0712.0207 [hep-ph]]. W. F. Chang, J. N. Ng and J. M. S. Wu, arXiv:0806.0667 [hep-ph] and arXiv:0809.1390 [hep-ph].

[36] K. Agashe, A. Delgado, M. J. May and R. Sundrum, JHEP 0308, 050 (2003) arXiv:hep-ph/0308036.

[37] K. Agashe, R. Contino, L. Da Rold and A. Pomarol, Phys. Lett. B 641 (2006) 62 arXiv:hep-ph/0605341.

[38] K. Agashe, R. Contino and A. Pomarol, Nucl. Phys. B 719, 165 (2005) arXiv:hep-ph/0412089; K. Agashe and R. Contino, Nucl. Phys. B 742, 59 (2006) arXiv:hep-ph/0510164; M. Carena, E. Ponton, J. Santiago and C. E. M. Wagner, Nucl. Phys. B 759, 202 (2006) arXiv:hep-ph/0607106 and Phys. Rev. D 76, 035006 (2007) arXiv:hep-ph/0701055; R. Contino, L. Da Rold and A. Pomarol, Phys. Rev. D 75, 055014 (2007) arXiv:hep-ph/0612048; A. D. Medina, N. R. Shah and C. E. M. Wagner, Phys. Rev. D 76, 095010 (2007) arXiv:0706.1281 [hep-ph]]; C. Bouchart and G. Moreau, arXiv:0807.4461 [hep-ph]; S. Casagrande, F. Goertz, U. Haisch, M. Neubert and T. Pfoh, arXiv:0807.4937 [hep-ph].

[39] V. Barger, T. Han and D. G. E. Walker, Phys. Rev. Lett. 100, 031801 (2008) arXiv:hep-ph/0612016; U. Baur and L. H. Orr, Phys. Rev. D 76, 094012 (2007) arXiv:0707.2066 [hep-ph]] and Phys. Rev. D 77, 114001 (2008) [arXiv:0803.1160 [hep-ph]]. 
[40] J. Thaler and L. T. Wang, JHEP 0807, 092 (2008) arXiv:0806.0023 [hep-ph]].

[41] D. E. Kaplan, K. Rehermann, M. D. Schwartz and B. Tweedie, arXiv:0806.0848 [hep-ph]; L. G. Almeida, S. J. Lee, G. Perez, G. Sterman, I. Sung and J. Virzi, arXiv:0807.0234 [hepph]; L. G. Almeida, S. J. Lee, G. Perez, I. Sung and J. Virzi, arXiv:0810.0934 [hep-ph].

[42] A. Pukhov et al., Preprint INP MSU 98-41/542; A. Pukhov et al., arXiv:hep-ph/9908288; A. Pukhov, arXiv:hep-ph/0412191.

[43] J. Pumplin, D. R. Stump, J. Huston, H. L. Lai, P. Nadolsky and W. K. Tung, JHEP 0207, 012 (2002) arXiv:hep-ph/0201195.

[44] L. March, E. Ros, M. Vos, "Signatures with multiple b-jets in the Left-Right twin Higgs model - fast simulation study of the ATLAS reach," talk presented at the Les Houches BSM working group, Twin Higgs discussion session, 23rd June, 2007.

[45] T. Sjostrand, S. Mrenna and P. Skands, JHEP 0605, 026 (2006) arXiv:hep-ph/0603175.

[46] D. Benchekroun, C. Driouichi, A. Hoummada, SN-ATLAS-2001-001, ATL-COM-PHYS-2000020, EPJ Direct 3, 1 (2001); W. Skiba and D. Tucker-Smith, Phys. Rev. D 75, 115010 (2007) arXiv:hep-ph/0701247]; B. Holdom, JHEP 0703, 063 (2007) arXiv:hep-ph/0702037 and JHEP 0708, 069 (2007) arXiv:0705.1736 [hep-ph]]; M. Strassler, "Unusual Physics Signatures at the LHC," talk presented at the 2007 Phenomenology Symposium - Pheno 07, University of Wisconsin, Madison, May 7-9, 2007; Gustaaf Brooijmans, talk at the "Workshop on Possible Parity Restoration at High Energy", Beijing (China) June 11-12, 2007; M. H. Seymour, Z. Phys. C 62, 127 (1994); J. M. Butterworth, B. E. Cox and J. R. Forshaw, Phys. Rev. D 65, 096014 (2002) arXiv:hep-ph/0201098; J. M. Butterworth, J. R. Ellis and A. R. Raklev, JHEP 0705, 033 (2007) arXiv:hep-ph/0702150; J. M. Butterworth, A. R. Davison, M. Rubin and G. P. Salam, Phys. Rev. Lett. 100, 242001 (2008) arXiv:0802.2470 [hep-ph]] and arXiv:0809.2530 [hep-ph].

[47] C. T. Hill and E. H. Simmons, Phys. Rept. 381, 235 (2003) [Erratum-ibid. 390, 553 (2004)] arXiv:hep-ph/0203079.

[48] G. F. Giudice, C. Grojean, A. Pomarol and R. Rattazzi, JHEP 0706, 045 (2007) arXiv:hep-ph/0703164.

[49] M. Perelstein, M. E. Peskin and A. Pierce, Phys. Rev. D 69, 075002 (2004) arXiv:hep-ph/0310039]; T. Han, H. E. Logan and L. T. Wang, JHEP 0601, 099 (2006) arXiv:hep-ph/0506313; R. Rattazzi, PoS HEP2005, 399 (2006) arXiv:hep-ph/0607058. 